\title{
DIE DIGBY-SPIELE.
}

(Fortsetzung und schluss.1)

\section{Maria Magdalena.}

Auf Saul's bekehrung folgt in der ausgabe der Digby Mysteries von Furnivall das mirakel Maria Magdalena. Seine quelle ist die bibel und die legende. Aber auch hier haben wir eine von anderen darstellungen unabhängige behandlung des lebens jener heiligen, im gegensatz stehend zu der gemeinsamen, aus der getreuen thbertragung des inhaltes der Legenda aurea entstandenen version der legenden, wie sie uns in Horstmann's ausgaben aus den jahren 1878, 1881, 1883 (Osbern Bokenam) vorliegen oder im Liber Festivalis des Johannes Myrcus enthalten sind; auch mit Caxton's Golden Legend, der sich, obgleich er sonst freier verfährt gegenttber der Leg. aur., gerade in der Mar. Magd. strenger an seine vorlage hielt, findet sich keine nähere ähnlichkeit; er hat keinen der abweichenden zthge, die unsere fassung charakterisieren. An die Legend dorée des Jean de Vignay als quelle ist wol kaum zu denken; sie ist ja uberdies nur eine ubersetzung der Leg. aur. ${ }^{2}$ Abgesehen davon, dass eine dramatische behandlung schon an sich manche änderung gebot, die in einer einfachen erzählung nicht nötig war, zeigt unsere fassung einzelne von der legendenversion abweichende punkte, die uns einerseits die selbständigkeit der produktion, andererseits in verbindung mit dem zusammenschweissen von mysterium, mirakel und moralität, die späte

1 Auf nachstehenden blïttern liegt der noch nicht gedruckte teil einer im jahre 1883 bei der universität Berlin eingereichten und von ihr gebilligten dissertation vor. Eine anzahl exemplare, den ersten teil (einleitung, Candelmes Day and the Kyllynge of the Children of Israell, The Conversyon of Seynt Paule) enthaltend, ist in der buchhandlung von Max Nicmeyer in Halle vorrätig.

2 Vgl. Dunlop, Prosadichtungen ed. Liebrecht s. 305. 
zeit der abfassung, in der man sich nicht schente, von der alten tiberkommenen form abzuweichen, anzeigen. Mit Wagner's jungerem stticke: The Life and Repentance of Mary Magdalene, gedruckt 1567, hat, nach Collier, History of Engl. Dram. Lit. II, 242 note 1, unser spiel, abgesehen von der gleichheit des stoffes, keine ähnlichkeit; tuberdies behandelt dieses das leben der heiligen nur so weit die bibel es erzählt und hat nichts legendarisches. ${ }^{1}$

Ward, History of Engl. Dram. Lit. I, 53 nennt dieses stick: 'by far the most remarkable', 'by far the most elaborate'. Ich glaube nicht, dass er viele anhänger seiner meinung finden wird; es ist meiner ansicht nach eine so schwache leistung, im anfange besonders zusammengewturfelt aus allen möglichen, yänzlich uberflissigen scenen, die auch dem dichter nicht einmal durch seine quelle geboten wurden, dazu des schmuckes entbehrend, der sonst z. b. die vorher besprochenen spiele nnserer sammlung auszeichnet: eines regelmässig durchgefuhrten kunstlichen strophenbaues, dass wir leicht erkennen, wir haben es mit der leistung eines auf dem gebiete der poesie gänzlich unbedeutenden mannes $z \mathfrak{u}$ tun. Um allem missgeschick die krone aufzusetzen, hat ein des dichters würdiger abschreiber das machwerk unter die finger bekommen, der daran nach herzenslust emendierte, sodass es jetzt in 'höchst unerquicklicher gestalt vorliegt.

1 Eine noch unedierte legende, ihrem inhalte nach zu urteilen sicher aus sehr später zeit, befindet sich im Harleian Ms. 6211; ich gebe, da sie durch ihre abweichungen interessant ist, kurz ihren inhalt, wie ihn mir herr Dr. Cohn auf meine bitte freundlichst aus der handschrift mitteilte. Teil I, fol. 56-75r: The life and death of S. Mary Magdalene Or her life in sin, and death to sin, enthält die beschreibung des Palace of Pleasure, des lebens der Mary Magdalene in demselben, ihrer schönheit und ihres falles. Darauf die schilderung des erwachenden gewissens, von dessen qualen sie unstät umhergetrieben wird. - T'eil II, fol. 76-92r: Mary Magdalene's death to sin Or her life in Righteousnesse: infolge eines erdbebens verfällt sie in bewusstlosigkeit, aus der sie durch Gottes hand erweckt wird. Es wird ihr vergebung fuir ihre suinden verheissen, wenn sie bereue. Eine silbertaube zeigt ihr den weg zum palaste der weisheit, von wo sie zur reue geleitet wird. Sie begibt sich dahin, wo Jesus mit den pharisäern weilt, und salbt ihm die füsse, worauf sie getröstet fortgeht. Nach der kreuzigung Jesu beweint sie seinen tod auf Golgatha, sucht sein grab auf und beklagt mit den jïngern den verlust des leichnams. Christus erscheint ihr und macht sie zur trägerin der tröstenden botschaft an die jünger. Da endet das gedicht mit den worten $\Delta \dot{\delta} \xi \alpha \tau \bar{\varphi} \vartheta \varepsilon \tilde{\varphi} .-$ Fol. 93 stehen noch drei lateinische gedichte von je vier distichen, die uiberschriften fiihrend: De Christo cum Simone Pharisaeo prandente et Mariam Magdalenam comiter excipiente. Ad Mariam Magdalenam. Ad eandem. 
Es wird eröffnet durch den kaiser 'tyberyus' (ev. Lucae ] III, 1), der in versen, die wol alliterierend sein sollen, ruhe grebietet; eine person 'Serybyl' beruhigt ihn. Collier, H. E.D.L. II, 234, vor dessen gänzlich falscher inhaltsangabe zu warnen ist, hält Serybyl für einen eigennamen; ich glaube eher, es bezeichnet sein amt und ist eine verwilderung ans dem in v. 114 in klarer form vorliegenden worte skrybe (scriba), welches auch da, wie hier, neben dem 'provost' genannt wird und in v. 33 und vor v. 41 auch in der form Syrybbe und Serybb vorliegt; vgl. ne. scribble.' . Das wort 'scribae' war ja den lesern der Vulgata als ubersetzung von $\gamma_{\rho} \alpha \mu \mu \alpha \tau \varepsilon \tau_{\zeta}$ des urtextes sehr geläufig. In der bedeutung 'secretair' kommt es auch vor z. b. in 'A new enterlude of godly queen Hester'; auch in den Coventry Plays XXIII begegnet es.

Der kaiser will, so fährt die darstellung fort, alle christen vertilgen; vorläufig begnügt er sich mit der vertilgung von 'wyn and spycys'. Darauf machen wir die bekanntschaft des Syrus, vaters des Lazarus, der Maria und Martha in seinem schlosse Magdalum.2 Wie diese angabe, so stammt auch die ganze errïhlung aus der Leg. aur., 'De sancta Mar. Magd.' - Nachdem Syrus, alliterierend, wie gewöhnlich, geprahlt hat, bestimmt er schon im voraus die art der verteilung seiner besitzungen nach seinem tode, wobei das schloss Magdalum an Maria fält (weshalb sie den beinamen Magdalena fuhrt). Die kinder sind hocherfreut uber die liebe des vaters, und auch hier schliesst ein solennes mahl, bestehend in 'wyn and spycys', die scene. Die wichtige christenfrage scheint den kaiser Tiberius aber auch uber tisch beschäftigt zu haben, wenigstens entsendet er

' $e$ in der ersten silbe verlesen aus $c$; $v$. 114, wo $k$ steht, war das nicht möglich. Die endung $\cdot y l$ in Serybyl verdankt ihre entstehung vielleicht(?) einer reminiscenz an die in den Chester Plays (Salutation and Nativity) und Towueley Mysteries (Processus Prophetarum) vorkommende Sybbyll (Sibilla). Wir hätten darin keine schlimmere form als etwa in den Pharasitles (Pharisaei) des 'Lazarus' der Chester Plays ss. 216. 218 oder dem Berlylinew (Bartholomaeus) der Coventry Plays s. 289, 'eyrodde' (Herodes) bei Sharp, Dissertation on the pageants or dramatic mysteries anciently performed at Coventry, 1825, 8.99, 'Nonceose' (nuncius) ib. 8. 99, oder unseren 'phelysofyr' nach v. 166.

2 In Furnivall's inhaltsangabe, die nach englischer art neben dem texte hergeht, wird vermutet, das schloss sei Bethanien, vgl. zu den scenen 2.4.8; aber $\nabla .59$ ist es ja genannt: this castel of Maudleyn, gleich Magdalum castrum der Leg. aur. Von Magdalena (gleich Maudleyn) kann doch diese benennung nicht etwa stammen, da es ihr ja erst im folgenden geschenkt wird. 
jetzt seinen boten zu Herodes mit der aufforderung, in den seiner herrschaft unterstellten ländern gehörig fur ruhe und ordnung zu sorgen. Herodes selbst, den wir in der folgenden scene persönlich kennen lernen, fuhrt sich in seiner bekannten unverschämten art, in alliterierenden versen, ein, uns unter anderem seine herrschaften anfzählend: Jerusalem, Alapye, Assye, Tyr, Abyron, Bergaby, Bedlem. Man braucht keine quelle zu suchen, aus welcher der dichter ersehen habe, dass Herodes besitzer dieser reiche war. Es liegt vielmehr im charakter der prahlerischen rolle des Herodes, möglichst viele reiche als sein eigentum sich zuzuschreiben. Der verfasser kramte daher die namen aller länder, die er mit Judäa im zusammenhange stehend glaubte, zusammen, um der prahlerei mehr nachdruck zu verleihen. Aehnliches begegnet sehr häufig in den sammlungen, z. b. in den Town. Myst., Magnus Herodes, ist dieser nach aussage seines Nuncius beherrscher von Tuskane, Turky, Alle Inde and Italy, Cecylla and Surry, From Paradyse to Padiva, to mownt Flascon, From Egypt to Mantua, unto Kemptowne, From Sarceny to Susa to Grece, Both Normandy and Norwa. - Noch mächtiger ist er in den Chester Plays: 'For 1 am kynge of all mankynde. I welde this worlde'. - Auch sonst begegnen ähnliche angaben, z. b. in dem von Furnivall, Early English Poems and Lives of Saints, publicierten Pilate XXIV, z. 62: the kyng Herodes, that was tho kyng by kynde | of the lond of ierusalem and of galilee and ynde. - Als ihn seine 'phelysophyrs' auf die herrlichkeit des verheissenen kindes aufmerksam machen und sein erseheinen durch bibelstellen ${ }^{1}$ belegen, wird er in die höchste wut versetzt, bis seine Milites ihn trösten und der brief des kaisers ankommt, den er weiterschickt an Pilatus. $\mathrm{Zu}$ diesem briefschicken, das hier eine nachahmung des späteren nach Christi tode (v. $1249 \mathrm{ff}$.) zu sein scheint, gab wol veranlassung der in den Evv. apokrypha erwähnte brief: Epistola Pontii Pilati quam scribit ad Romanum imperatorem [Tiberium Caesarem] de domino nostro Jesu Christo. ${ }^{2} \mathrm{Zu}$ erinnern ist auch daran, dass in den Town. Myst., Caesar Augustus, der Imperator, von seinem Consultus von Christi erscheinen benachrichtigt, zu seinem vetter Syryn um rat schickt. - Schon in

1 v. 175/6: Jesaias 60, 3 ; $\nabla .184 / 5$ : Genesis 49, 10 .

2 Bei Tischendorf s. 431. 
dem alten 'Ludus Paschalis de adventu et interitu Antichristi' (12. jahrhundert) geschieht die verhandlung zwischen den auf ihren tronen sitzenden firsten nur durch boten.

Pilatus nimmt, nachdem er sich, in alliterierenden versen, eingefuhrt hat, den brief entgegen und schwört beim Mars (be Martes, v. 257; auch in den Chester Plays, Balaam and his asse, s. 81, ruft könig Balake den 'mightie Marse' an), fur aufrechterhaltung der gesetze sorge tragen zu wollen. - In der folgenden scene stirbt Cyrus, von seinen kindern gebtlhrend beweint, die seine seele dem 'inwytlissymus god' empfehlen.'

Der verfasser hatte nun gemäss der Leg. aur. zu schildern, wie Maria 'deliciis corporis se totam' hingab, und das gibt ihm gelegenheit, ein stilck moralität einzupflechten, indem er darstellt, wie die teufel eine beratung abhalten, auf welche weise sie Maria zu falle bringen können, da sie, wenn sie in der tugend beharrt, die hölle zerstöre. Dieser zug erinnert an 'The Develis Parlament', ca. 1430, ed. Furnivall, Hymns to the Virgin etc., s. 41; vgl. auch Cov. Plays, The Temptation, die beratung der teufel tiber Christus. - Daranf wird sie von ihnen in ihrem schlosse belagert. Auch das ist ein beliebter zug, der quellen genug bietet; z. b. vgl. den zweiten teil des Romans von der Rose: Amour belagert den turm, in dem Bel-Accueil sitzt, oder, und das ist wichtiger fur uns, da unsere darstellung nur nachahmung davon zu sein scheint, vgl. die moralität 'Castel of Perseverance', in dem die sieben todstinden das humanum genus in eben dem schlosse belagern. Nach Collier, H. E. D.L. II, 286 gehört dieses stllck der regierungszeit Heinrich's VI. (1422-1461) an, könnte also unserem dichter vorgeschwebt haben; beide verwenden, um auf diesen punkt noch aufmerksam zu machen, Luxuria, um den helden oder die heldin sicher zu falle zu bringen, und in beiden - vgl. v. 735 bei Furnivall and Collier a. a. o. II, 283 - werden die teufel fur ihre nachlässigkeit, sich eine seele haben entgehen zu lassen, von ihrem beherrscher beschimpft und geschlagen. Auch an das Grosseteste zugeschriebene 'Castel d'amour' und an das 'Castel of Labour', 'le Chateau de labour', nach Warton-Hazlitt III, 166 aus der regierungszeit Heinrich's VII., 1485-1509, sei erinnert.

1 Gemeint ist wol invictissimus, kaum, wie Furnivall glaubt, infinitissimus; vgl. Leg. aur., De $\mathbf{S}^{0}$. Paulo apostolo: 'nam et nos illi_regi invictissimo [Christo] militamus,' 
Wie schon erwähnt, bahnt sich Luxuria einen weg ins schloss, während die anderen laster nach Jerusalem gehen, wo wir sie später wider brauchen werden. Allegorische figuren begegnen auch in den sammlungen, z. b. Ludus Coventriae, 'Parliament of Heaven', 'Salutation and Conception'. Luxuria schmeichelt sich bei Maria ein, rät ihr, sich tber den tod ihres vaters dadurch zu trösten, dass sie sich den vergntigungen der welt hingibt, und gewinnt sie so für seinen vorschlag, dass sie sofort von bruder und schwester abschied nimmt und beiden ihr schloss thergibt. Es ist das eine vereinfachung der fassung der Leg. aur., wo zu gleicher zeit auch Lazarus seine heimat verlässt, um soldat $\mathrm{zu}$ werden, und daher beide ihrer schwester Martha ihre besitzungen anvertrauen. Luxuria und Maria gehen nach Jerusalem zu einem 'Tuverner', wo sich denn auch bald ein 'galaunt', 'Coryoste', einfindet, dem Luxuria sein opfer getrost tuberlässt. Die teufel aber äussern eine diabolische freude uber das gelingen ihres planes. Alles das ist ausmalung der aussage der Leg.aur.: cum autem Magdalena deliciis corporis se totam exponeret etc., und: corpus suam voluptati subtraxit.

Doch die erlösung vom tbel naht. Simon der aussätzige erklärt seine absicht, zu dem mahl, das er für seine freunde bereitet hat, auch den propheten (Jesus) einzuladen (Lucas VII, 36 in verbindung mit Marcus XIV, 3). Da gemäss der bibel bei gelegenheit dieses mahles die absolution Maria's stattfand, so batte der dichter sie darauf vorzubereiten, und er tat dies unter benutzung zweier äusserungen der Leg. aur.: illa aulem nutu inspirata divino - und später: dominus ad poenitentiam ipsam misericorditer convertil, inden ihr guter engel sie auf ihr stundiges leben aufmerksam macht. Auch in den deutschen stticken ist diese art, die stinderin vom bösen abzubringen, gewöhnlich. ${ }^{1}$ Zum gltick schenkt sie dieser stimme gehör und beschliesst, zu ihrer inneren heilung den propheten aufzusuchen, welcher, der einladung Simon's folgend, in dessen hause weilt. Dort wäscht sie seine fusse mit ihren tränen, trocknet sie mit ihren haaren und salbt sie (vgl. Luc. VII, 37/8; näher liegt noch die darstellung der Leg. aur., wo, wie bei uns, das kttssen der ftusse nicht erwähnt ist gegenuber der schilde-

' So in dem bei Mone, Schauspiele II, 190, Hoffmann, Fundgruben iI, 248 stehenden oder in dem von Haupt edierten 'bruchstilck eines osterspiels' im Archiv für die deutsche Sprache und Dichtung von Wagner I, 355. 
rung des Lucas). Christus dankt Simon, hat ihm aber noch etwas zu sagen (Luc. VII, 40). Dann kommt das gleichniss von den beiden schuldnern, deren schulden bei uns 100 und 50 'pense' betragen, aber in Chest. Plays gleich der Vulgata 500 und 50 (Lnc. VII, 41/3; vgl. \%.661: Recte judicasti, aus der Vulgata ubernommen). Es folgt die anwendung des gleichnisses auf den vorliegenden fall (vv. 44 und 46), erwähnung seiner gedanken uber Jesus (nach v. 39) und vergebung der sünden der Maria (v. 48), die nun in jubel ausbricht, Jesum ihre medizin nennt' und besserung yelobt. Darauf die erwiderung Jesu: 'thy feith hath savyt the' (fides tua the salvam fecit) und 'Vade in pace' (v. 50). Dann treibt er ihr die sieben teufel aus (Leg. aur.: $a b$ ea septem daemonia expulit), der gute engel freut sich uber Maria's bekehrung und bittet um Jesu schutz, während der Rex diabolus mit zwei genossen: Belfagour und Belzabub (dem Belphegor und Beelzelbub der Vulgata) gericht hält tber die sieben bösen geister der Maria und sie zthehtigt. Die bekehrte selbst aber kehrt zu ihren geschwistern zurtlck und wird mit grosser freude aufgenommen, einer freude, die leider dadurch jähe getrtibt wird, dass ihr bruder Lazarus krank wird (Joh. XI, 1.2). In ihrer not beschliessen sie, za Jesus, der ihn ja liebt, zu eilen und ihn um hilfe zu bitten (ib. v. 3. 5; in der bibel schicken sie zu Jesus). Jesus tröstet sie: 'of all infirmite ther is non to deth' (infirmitas haec non est ad mortem, v. 4) und kntipft daran noch für uns unverständliche betrachtungen uber die heilige dreieinigkeit. Darauf stirbt Lazarus ('mortuis est') und wird unter grossem trauergefolge (vgl. v. 19) begraben. Erst jetzt macht sich Jesus mit seinen begleitern (er nennt sie 'chyldryn of lith' nach v. 9/10) auf nach Judäa (v. 6/7), um Lazarus aus dem schlafe zu wecken (v. 11). Fin dissipulus (bibel: discipuli) spricht seinen glauben ans, dass Lazarus trọtz seines schlafes doch gerettet werden könne (missverständlich nach v. 12: si dormit, salvus erit), während Jesus von dessen tode gesprochen hatte (v. 13: dixerat autem Jesus de morte ejus). Unser dichter dagegen, dem die regel tuber die verschiedenen arten, das possessivpronomen der dritten person

1 Vgl. Town. Myst., Resurrectio Domini s. 262: for to eche sore he was medecyne. Henderson, Missale Ebor.II, 215: medicina nostra in der Sequentia; ebenso s. 243 u. ̈̈. Vgl. auch Konrad von Wiirzburg, Goldene Schmiede, ed. W. Grimm, LI: 'weil C'hristus uns von der krankhcil der sünde heilt'.

Anglia, VIII. band. 
im Lateinischen auszudrticken, je nachdem es reflexiv ist oder nicht, jedenfalls nicht recht klar war, glaubt, er habe tiber seinen eigenen tod gesprochen, und lässt Jesus daran eine betrachtung über seine mission knüpfen. Er fährt dann fort, Lazarus sei wirklich gestorben (v. 14), und es freue ihn, dass dieser umstand mit zur erhöhung ihres glaubens beitragen könne (v. 15). Ein jude (entnommen aus v. 31) meldet seine ankunft. Martha läuft ihm entgegen mit der versicherung, ihr bruder wäre nicht gestorben, wenn er dagewesen wäre (v. 21); aber er versichert ihr, ihr bruder werde wider anferstehen (v. 23), nicht bloss am jungsten tage, wie sie zugibt (v. 24), sondern sofort durch den glauben an ihn (v. 25/6). Maria kommt dazu (v. 29), fällt Jesu zu füssen, auch unter der versicherung, seine gegenwart würde den tod abgehalten haben (v. 32). Jesus lässt sich seine grabstätte zeigen: where have you put him? (ubi posuistis eum, v. 34), der stein wird abgenommen (v. 39.41), er betet zu Gott (v. 42) und ruft Lazarus zu sich ( kommt in seinem leichengewande (v. 44). Alles volk aber ist von solcher tat natturlich therzeugt und glaubt an Jesum (v. 45).

Wir tuberspringen jetzt die zeit der passion Christi; die folgenden scenen fthren uns in den gang der begebenheiten nach seinem tode. Erst machen wir noch die bekanntschaft des 'kyng of Marcylle' (des 'princeps' der provinz 'Masillia' nach der Leg. aur.), der uns, naturlich prahlend und in alliterierenden versen, sich und fran gemahlin, letztere als ausbund von vortrefflichkeit vorstellt, worauf sie ihm ihren dank abstattet; auch diese zarte familienscene findet in einem fröhlichen mahle mit den unvermeidlichen ' $w y n$ and spycys' ihren abschluss.

In der folgenden scene macht ein teufel, der mit dem gewöhnlichen rufe: 'owyt, owyt, harrow' erscheint (vgl. Sharp, Dissertation s. 59), uns mitteilung von der eroberung der hölle durch Christum. Seine schilderang bewegt sich in den gewöhnlichen ausdrticken, wie sie aus dem Ev. Nicodemi, Descensus Christi ad Inferos in die Leg. anr. etc. thergiengen, ohne sich aber entfernt so eng an die quelle anzuschliessen, wie etwa das sttck 'Harrowing of Hell' in den Chester Plays; anklänge an das gedicht 'The Harrowing' of Hell' finden sich nicht.'

1 Vgl. z. b. v. 966: ower barres of iron are all tobrost! strong gales of brass! mit der aufforderung des Infernus - in Tischendorf's ausgabe text A, kap. V - claudite portas crudeles acreas et vectes ferreos sup. 
Unter benutzung solcher geläufigen vorstellungen schildert der teufel die tiitigkeit Christi nach seinem tode. Dies leitet uns tiber zu der erscheinung am grabe, die in gewöhnlicher weise geschildert wird: die drei Marien erscheinen an der heiligen stiitte (Marc. XVI, 1), sich mit schmerzen der einzelnen scenen der passion erinnernd, wie der gekreuzigte die weiber Jerusalems anredete (Luc. XXIII, $28 \mathrm{ff}$.), das kreuz fallen liess (nach Luc. XXIII, 26), wie man ihn verspottete (ib. 35); sie wollen seinen körper balsamieren; plötzlich erscheinen ihnen zwei engel (Luc. XXIV, 4) und ermutigen sie. ${ }^{1}$ Die frauen suchen Petrus und Johannes auf (Joh. XX, 2), teilen ihnen die abwesenheit des leichnams mit, und alle machen sich auf den weg zum grabe (Joh. XX, 3). Dort finden sie das schweisstuch des toten (Joh. $\mathrm{XX}, 7)$, erinnern sich seines versprechens, am dritten tage wideraufzuerstehen (Matth. XVI, 21 ff.), die ubrigen gehen fort, nur Maria bleibt - so mtissen wir ergänzen nach Joh. XX, 10 und dem sonst unverständlichen zusammentreffen Jesu mit ihr allein; die stelle ist wol litckenhaft, wenigstens in den buhnenweisungen, tuberliefert. ' $I$ us angelus' - also sind gemäss Joh. $\mathrm{XX}, 12$ zwei engel gemeint - fragt sie: woman, woman, wy wepest thou? (mulier, quid ploras? v. 13). Sie antwortet, sie suche den leichnam Christi; dieser erscheint ihr selbst (v. 14), fragt sie: woman! woman! wy syest thou? wom sekest thou? (mulier, quid ploras? quem quaeris? v. 15), sie erkennt ihn nicht, fragt auch ihn, ob er etwas wisse um ihren herrn, bis er sie anruft: 0 Mari (Maria, v. 16), sie erkennt ihn: A, gracyous master and lord! (Rabboni, quod dicitur magister, v. 16) und will ihn ktssen (ebenso wird in den Town. Myst. das unbegrtindete Noli me tangere der bibel gerechtfertigt). Aber er ent-

ponite! und der schilderung in text B, kap. VIII: subito infernus contremuit et portae morlis et serae comminutae et vectes ferrea confracti sunt et ceciderunt in terram et patefacta sunt omnia; oder the kyng of Joy Leg. aur., De Resurrectione Domini: Rox gloriae - enteryd ... as bryth as fyrys blase mit der stelle: subito illuxit nobis lux magna, B, II. Ebenso: Adam and Abram and alle hir kynred ongt of oner preson to joy were they take mit: Pax tibi [Adam] cum omnibus filiis tuis, A, VIII, und Jesus ... firma ima carcerum confregit et .... solvit vinctos et introduxit cos in paradisum - Leg. aur., a. a. 0.

1 'dredylt you rith nowth' (nolite expavescere, Marc. XVI, 6), 'Jesus is resun and is nal here' (surrexit non est hic), 'loo! here is the place that he was in browth' (ecce locus ubi posuerunt eum), 'go. sey to his dysypylles and to peler, he xall apere' ( 7 . 7 : sed ite, dicite discipulis ejus et Petro), 'in Galelye, ther xall ye se him, lyke as he said' (quia praecedit vos in Galileam, ibi eum videbitis, sicut dixit vobis). 
zieht sich ihr: Towche me natt, Mary! I ded natt asend To my father in deyyte, and onto yovers (Noli me tangere, nondum enim ascendi ad patrem meum) But go, sey to my brotheryn, I will pretende to stey to my father in heunly towers (vade antem ad fratres meos et dic iis: ascendo ad patrem meum et patrem vestrum). Maria erzählt ihm, sie habe ihn zuerst für den gärtner gehalten (nach v. 15), und Jesus benutzt ihren irrtum, sich als gärtner der menschenseelen zu bezeichnen, eine wendung, die keine der sammlungen hat. Dann, nach einer ermahnung an sie, verschwindet er; sie aber macht ihrem freudegeschwellten herzen dadurch luft, dass sie ihren genossinnen die begebenheit erzählt (nach v. 18); zusammen eilen sie dann, es den juingern mitzuteilen (Matth. XXVIII, 8). Auf diesem wege begegnet ihnen Jesus, redet sie an: Avete (Avete, Matth. a. a. o. 9), segnet sie and gibt ihnen den anftrag: go ye to my brelhyrn and sey to hem ther, that they procede and go into Galelye; and ther xall they se me (ite, nunciate fratribus meis, ut eant in Galileam, ibi me videbunt, v. 10), darauf verschwindet er.

Die folgende scene ist dem ergötzen gewidmet; es ist ein gottesdienst, dem Muhamed zn ehren, zu dem sich der könig of Marcylle mit seinem gefolge begibt, 'to do a sacryfyce'. Zu dieser scene wurde der dichter durch die Leg. aur. veranlasst, wo es heisst, Maria habe in Marseille das volk zum tempel eilen sehen: ut ydolis immolaret. Die einleitung zum gottesdienst besteht in einer reihe unsauberer scherze, schimpfreden und schlägen zwischen dem 'presbyter' und dem 'clericus' 'Hawkyn'. Solche namen aus dem gewöhnlichen leben begegnen öfter.' Die vorlesung bildet eine reihe von zeilen im kauderwälsch, lateinische endungen tragend, nach Warton-Hazlitt III, 287 note 3 in dem metrum und der art Skelton's, unter anrufung von Ragnell und Ruffyn, beide bekannt aus den Chester Pl., wo im 'Antichrist' ein teufel Ragnell heisst, während im 'Fall of Lucifer' Ruffyn ein gefährte des Lucifer ist; in Coventry Pl. begegnet dieser name hänfig.

In der folgenden scene kehrt dann das schon einmal beobachtete briefliche benachrichtigen der fürsten, diesmal tiber Jesu tod, wider. Pilatus beratschlagt mit seinen 'serjauntes';

2 Z. b. in Coventry Pl., Parliament of Heaven, zum schluss eine ganze reihe; Town. Myst., Secunds Pastorum, heissen die hirten: John, Horne, Parkyn, Gybon Waller, Mac; in der Prima Pastorum Jack etc 
die auferstehung Jesu hat eindruck auf ihn gemacht; trotzdem das grab besetzt worden ist (Matth. XXVII, 66), ist er doch auferstanden; 'Joseph of Baramathie' (Arimathia) batte den leichnam an sich genommen (Matth. XXVII, 57 u. ö.). Die serjaunles meinen, unan müsse es so darstellen, als hätten seine schtler ihn gestohlen (Matth. XXVIII, 13), und das solle Pilatus auch dem kaiser mitteilen. Demgemäss sendet er seine boten mit diesen nachrichten zunächst zu Herodes, der sich freut, nun mit Pilatus wider ausgesöhnt zu sein, während sie vorher feinde waren (Luc. XXIII, 12), worauf der bote seinen weg zum kaiser fortsetzt. ${ }^{1}$

Jetzt kehren wir wider zu unserer heldin zurtick; sie erinnert sich schmerzerfullt der vergangenen ereignisse, des todes Jesu, der auferstehung, der glossolalie der jtinger (Act. App. II, 6) und ihrer zerstreuung in alle länder, um das heil zu predigen (Leg. aur.: Post adscensionem igitur Domini ... diversarum gentium discipuli subeunt regiones, verbum domini ibidem seminantes). Begleitet ist sie von ihrem 'dysypyll' (Maximinus nach der Leg. aur.: erat autem tunc temporis cum apostolis beatus Maximinus, unus de 72 domini discipulis, cui a beato Petro Maria Magdalena fuerat commendata). Nach einer verherrlichung der jungfrau Maria durch Jesum in der gewöhnlichen art², schickt der herr der Maria durch Raphael den befehl, 'Marcyll' (Leg. aur.: Massilia) zu bekehren. Hierin wich der dichter von seiner quelle $a b$, wo erziahlt wird, Maria sei mit genossen von einem heidenvolke auf ein schiff gesetzt, sei aber 'divino tandem nutu' nach Massilia gekommen. Aber die darstellung einer solchen fahrt, tuberflussig wie sie war, war dramatisch ja unmöglich und so verwendete er denn den 'nutum divinum' in derselben weise, in der Saulus von den ihn bedrohenden nachstellungen benachrichtigt wurde, durch den engel, und liess die heldin in ganz gewöhnlicher weise nach Massilia kommen, indem sie sich von einem schiffer übersetzen lässt, was um so näher lag, als wir ganz dieselbe scene naturgemäss sich nach-

1 Vgl. den oben erwähnten apokryphen brief und ebenso den im ev. Nicodemi erwïhnten, welchen Pilatus an den könig Claudius in derselben angelegenheit schickt.

2 Vgl. z. b. bei Furnivall, Hymns to the Virgin and Christ s. $1 \mathrm{ff}$; zil 'fles of Judcon' z. 1351 vgl. Henderson, Missale Ebor. II, 208: ave... vc:llus Gedeonis; empress of hell, z. 1359, begegnet auch bei Furnivall, Political etc. Poems 81, 3. 
her bei der fahrt des königs werden widerholen sehen, und auch in der fassung der so verwanten sage der Maria Aegyptiaca - wir werden noch eine beeinflussung beobachten - sich diese einfache art findet. Angekommen begibt sie sich sofort zum könige, mit dem sie denn auch gleich in einen, natturlich erfolglosen, streit tuber ihren glauben gerät.' Doch interessiert er sich ftir ihren gott wenigstens insofern, als er sich von ihr die schöpfungsgeschichte erzählen lässt, die sich nach der einleitung: In principio erat verbum (Joh. I, 1) an die in der Genesis gegebene schilderung anschliesst. Um ihr einen begriff von der macht seines gottes zu geben, nimmt er sie mit in den tempel und bittet Muhamed, zunı zeichen seiner herrlichkeit zu sprechen. Aber vergeblich. Mehr erfolg hat ein gebet der Maria, denn nachdem sie es gesprochen 'xall the mament trembyll and quake'. Dieser zug, der sich in der Leg. aur. nicht findet, scheint veranlasst durch die stelle: cum aulem quadam die Maria Magdalena praedicaret, praedictus princeps dixit ei: 'putas posse defendere fidem, quam praedicas?' cui illa 'equidem illam defendere praesto sum, utpote quotidianis miraculis ... corroboratam', indem der dichter entweder die participialkonstruktion nicht verstand und sich nur an das 'miraculis' hielt, oder aber indem er es in freier weise behandelte - fur beides haben wir ja schon beispiele gefunden; jedenfalls schwebte ihm wol das wunder beim betreten Aegyptens durch die heilige familie vor (vgl. sttick I). Als darauf sogar der tempel durch ein feuer von oben in brand gesteckt wird, ist der heide noch nicht uberzeugt, sondern verspricht ihr, sich die gelegenheit zu nutze machend, nach den worten der Leg. aur.: ecce dictis tuis per omnia obtemperare parali sumus (er und sein weib), si a deo quem praedicas nobis filium impetrabis, denn 'my wyff and I together many yeres have byn and never mith be conceyvid with child'.

Trotz ihres verkehrs mit einer so hochgestellten persönlichkeit ist aber fur Maria's unterhalt noch nichts geschehen, und so wendet sie sich im gebet an Jesum, der ihr zwei engel sendet, mit denen sie in der nacht zum könig geht, den sie auffordert, der an ihn ergangenen warnungen zu gedenken (nach der Leg. aur. finden drei traumerscheinungen statt, und zwar

1 Vgl. Leg. aur.: cui [principi provinciae illius] Magdalena Christum praedicans sacrificia dissuasit. 
hat die königin sie; daran dachte der dichter, obgleich er vereinfacht) und sie zu unterstutzen. ${ }^{l}$ Die erscheinung hat den gewtinschten erfolg. (Guapropter ipsos hospitio receperunt et iis necessaria ministraverunt. Leg. aur. - In betreff der schilderung vgl. zu v. 1629 Leg. aur.: ac si tota domus arderel; zu v. 1630: vultu igneo; zu v. 1631: irata etc.). Der wunsch des königs scheint in erfüllung zu gehen, und so bekennt er sich zum glauben an Gott und unternimmt eine reise nach Jerusalem zu Petrus, um sich von diesem taufen zu lassen (nach Leg. aur.: um zu sehen, ob sich alles wirklich so verhalte, wie Maria ihm geschildert hat). Auch die königin wird auf ihre bitte mitgenommen und Maria zur viceregentin eingesetzt. ${ }^{2}$ Beide machen sich auf den weg. Hier, wie auch bei der tuberfahrt der Maria vorhin, findet eine scene zwischen dem schiffer und seinem jungen zur erheiterung statt. Auf ihrer fahrt thberfallt sie ein sturm; die königin, scheinbar tot ${ }^{3}$, wird mit ihrem kinde auf einen felsen ausgesetzt, die fahrt darauf glucklich beendet. Der könig trifft auch sofort Petrus (Leg. aur.: Petrus ei obvius fuit), wird von ihm getauft, bleibt zwei jahre bei ihm, besucht die heiligen orte, fährt zurtick, findet frau und kind lebend auf dem felsen wider, hört, dass auch die frau in Jerusalem war u. s. w., sie kehren in die heimat zurttck - alles das genane widergabe der Leg. aur.

Als sie zu hause ankommen, finden sie Maria predigend. (Leg. aur.: invenerunt beatam Mariam Magdalenam cum suis discipulis praedicantem.) Sie ermahnt zur beständigkeit im glauben, auch wenn man zuweilen in armut sei (naheliegend bei dem vorher ausgestandenen mangel); dem schliessen sich dann noch vorschriften an, die der bergpredigt nachgebildet sind (vgl. z. 1930/1 und Matth. V, 3; z. 1932 und v. 4; z. 1933 und v. 5; z. 1934 und v. 6 ; z. 1935 und v. 7). König und königin fallen vor ihr nieder (Leg. aur.: ejus pedibus cum lacrymis provoluti) and danken ihr,

1 Furnivall's inhaltsangabe der buhnenweisung nach z. 1618 ist falsch. Der engei und Maria tauschen das vorhin angezogene gewand jetat nach der traumerscheinung wider gegen ihr gewöhnliches um; vgl. vv. 1605 . 1608. 1624. - Geringere versehen finden sich in der inhaltsangabe der vv. $1412 / 4$ und 1478 .

2 In Leg. aur. wird bei dem entschlusse des königs, die fahrt zu unternehmen, zuerst der königin dic stellvertretung übertragen, und erst als diese erkliirt, teilnehmen zu wollen, der Maria; bei uns ihr sofort mit umgehen der königin.

3 Furnivall's inhaltsangabe 'and dies' ist nicht richtig. 
sie aber geht sofort in die wiste 'more gostly strenkth me to purchase' (supernae contemplationis avida). Der könig aber baut kirchen (Christi ecclesias construxerunt) und entsagt dem Muhamed (Leg. aur.: omnium ydolorum templa destruentes).

In der wtiste wird Maria auf Jesu befehl von engeln, die sie in die wolken erheben, mit himmlischem brote gespeist (Leg. aur.: redemptor noster ipsam non terrenis refectionibus sed tantum coelestibus epulis disposuerat satiare). ${ }^{1}$ Es geschieht dies unter dem gesange der engel: Assumpta est Maria in nubibus; coeli gaudent, angeli laudantes felium Dei. ${ }^{2}$

So findet sie einst ein priester, der ebenda in der witste sich aufhält; sie erzählt ihm, sie sei schon dreissig jahre dort und werde täglich dreimal von oben gespeist (aber Leg. aur.: septenis vicibus per singulos dies). Leg. aur. erzählt dann weiter, dass Maria dem priester aufträgt, zum bischof Maximinus zu gehen und ihm zu melden, dass sie am nächsten auferstehungsfeste in seiner kirche erscheinen werde und er ihr dann das heilige abendmahl reichen solle. Unser dichter dagegen hat, anknupfend an ihren ausspruch gegentiber dein priester in der Leg. aur.: 'Quia igilur mihi a domino revelatum est, quod ex hoc migratura sum seculo etc.', uns geschildert, wie diese enthtllung geschah, auch hier wider durch vermittelung zweier engel, deren einer der Maria die mitteilung macht, dass sie wegen ihrer frömmigkeit die krone des sieges erhalten und ganz in den himmel erhoben werden solle, während der andere den priester auffordert, der Maria das heilige abendmahl zu reichen, also mit umgehung des bischofs (der z. 2129 erwähnt wird), wie auch in der Maria Aegyptiaca der priester selbst dies amt verrichtet. Nach dem genusse des abendmahls stirbt sie unter der frende der himmlischen (gaudent in celis).

Den epilog spricht der priester, z. 2132-36; z. 2137-40, wo nochmals derselbe gedanke kommt: 'thus endytt the sentens (thys mater), schliessend mit der gewöhnlichen aufforderung, das Te Deum zu singen ${ }^{3}$, scheint später hinzugedichtet zu sein, viel-

1 In Furnivall's inhaltsangabe der vv. $2012 \mathrm{ff}$. ist ein fehler: Jesus wird von den engeln gepriesen, nicht Maria.

2 Vgl. die 'Versus in die Assumptionis beatae Mariae' bei Henderson, Missale Ebor. II, 81: Assumpla est Maria in caelum, gaudent angeli et collaudantes benedicunt Dominum.

3 Vgl. Hase, Das geistliche Schauspiel s. 51. 
leicht von dem schreiber, der auch die zz. 2141/4 hinzusetzte. So ist leicht verständlich, dass er sich z. 2143 an die 'redars' wendet, während eben vorher, z. 2133, von dem spielen des stickes gesprochen wurde. Aber zweifelhaft ist auch, von wem das 'Explicit oreginale de suncta Maria Magdalena' herrtihre; entweder war damit das original, das dem dichter vorlag, also die Leg. aur., oder das original, von dem der kopist seine abschrift nahm, gemeint. Jedenfalls aber scheint mir der begriff 'original' festgehalten werden zu mulssen, und ist wol kaum daran zu denken, wie Collier, H. E. D. P. II, 231, es auffasst, dass dies der titel unseres stlickes sei.

Die sprache des dichters ist die des westlichen mittellandes; das wird bewiesen durch den plur. ind. praes. -n: desyern 721 (: etern), gon 1234 (: bon), byn 1533 (: agayn), so auch noch oft innerbalb des verses, z. b. 16. 40.50 etc., und durch 3. sing. praes. ind. -s: daunnes 35 (: lawys), anch telles : rebelles : dwelles 123 spricht dafur, da wir hier die alten urspriinglichen formen im reime, wo ja die schreiber sorgfältiger waren, erhalten haben gegenüber den innerhalb der verse vorkommenden formen -th: doth 122, holdyth 126 etc. Innerhalb des verses begegnet -s nur noch sehr selten, z. b. bryngis 18.

Gegen den norden spricht das im part. praet. starker verba oft abgeworfene -n: draw 236 (: law), be 642 (: the), 892 (: equite), take 978 (: wrake) etc. Ebenso der iibergang eines ae. â zu ô: bold 147 (: world), most 632 (: trost), soo 830 (: doo), more 1202 (: pore). Wenn aber daneben auch noch das alte â erhalten vorkommt - wenigstens zeigt das der reim, wenn auch der schreiber diesen verdorben hat, indem er nach seinem südlichen dialekt o setzt - , so zeigt uns das, dass wir es ebenso wenig mit einem siidlichen denkmal zu tun haben, sondern dass dieses dem mittellande, wo beide formen sich begegnen, angehört; so z. b. findet sich mare 60 (: care), 1537 (war), sare 63 (: dare). - Gegen den silden spricht auch die entwicklung eines ae. y als umlaut zu älterem u, o zu i: fyr 597 (: ire), pryde 35s (: tyde), synne 378 (: wynne), kysse 571 (: prysse) etc.

Wenn wir neben diesen formen andere finden, die $\mathrm{zu}$ ihnen im gegensatze stehen, so haben wir darin vom schreiber eingefuhrte formen zu erkennen; dieser aber hatte kentischen dialekt. Daher zeigt der ind. praes. plur. neben der schon erwähnten und der flexionslosen form auch das südliche -th, z. b. doth 43. 459, seyth 64i etc. Sehr selten -t: devydytt 955. Wenn wir diese endung -th auch einmal am ende der zeile im reime finden: beth 1528 (: hed), so ist auch das dem kopisten zuzuschreiben, der dem reime be : hed aufhelfen wollte.

In der 3. sing. ind. praes. findet sich innerhalb des verses gowöhnlich die endung -th, auch -t: stondyt 6, commyt 17, growyt 20 etc.

Einzeln finden sich noch die ae. formen fur 3.plur. pron. pers. neben den gewöhulichen nürdlichen formen: hem 40. 91.119 etc., he 366. 370, here 1124, nach 1618. 
Für ae. $y$, umlaut eines älteren $u$, o, findet sich innerhalb der verse sehr oft das kentische e; so knett 58, dent (ae. dynt) 272, frest (fyrst) 971, nach 739; frett (ae. fyrhto) 786, shert (an. skyrta) 496, berdes (ae. brŷd) 51, felle (ae. fyllan) 535, kendnesse 1342 etc. - Ebenso ist kentisch die vorliebe für e überhaubt, so bei uns statt i, z. b. lem (ae. lim) 13, shep 1351, whech (8e. hwilc) 183, drevyn 1955, wett 1945, rese (ae. rîsan) 180, then (ae. thin) 1732, smet (ae. smiten) 519, settyng 361, lekeyng 1132, abedyn 1678, led (ae. hlid) 1015, better 666, ded 733, leffe 766, therd 809. - Ebenso in rom. worten: merrorys (afrz. mireor) 73 , pete 239, mesteryys 2041, serys 418, ternite 718, esperyt 733, phelysofyr nach 161 , perelle (wo der reim perille verlangt) 1916. - Auch in lat. worten: sene 1972, speritu 1862, vergo 1900 , felium 1120, domenicum nach 2191 , oreginale nach 2140 , deabolus nach 721 .

Bei der vorliebe, die der spätmittelkentische dialekt hat, z für anlautendes $8 \mathrm{zu}$ setzen, ist man wol geneigt, einige fälle hier anzuführen, in denen $z$ fuir $\mathrm{s}$, freilich nicht anlautend, sich zeigt; es kommt so geschrieben auch nur einmal vor: pleze 1548; aber nicht selten finden wir 3 , wo wir s oder nach obiger art $z$ erwarten, beruhend also auf einem fehler des schreibers, der beide buchstaben verwechselte, wie er es auch tat in Belzabub 725, Berzaby 159 etc., so finden wir von obigem worte auch die form pleze 659, und in demselben wortstamme: plezauns 462. 1304, plezaunt 957. 1540, plezing 1491. 1504. 1646 etc. Dagegen folgte er gewöhnlich der vorlage und schrieb s: plesorvans 90. 100. 355 etc., plesaunt 349, plesyng 1480 .

Der ungelibte schreiber verrät sich auch noch sonst; er verwechselt ebenso $\zeta$ und P: zese 555, $\zeta^{e}$ (artikel) 584. 664. 1251 . (pron. pers.) 593. 1740. 1841, zether (ae. pider) 1882 etc. Und umgekehrt $p e$ fir ze 925. 1433.

An orthographischen eigentümlichkeiten sei erwähnt: Berechtigtes (g)h fehlt: thow (ae. pêah) 203, browt (brought) 208, dowller (dohtor) 99, auch dowctor 68. 416 oder docclor 877, myty (mihtig) 12, reylyus (rihtwis) 212, whytly (wiht) 376 , syte (siht) 554, knytes (cniht) 50,

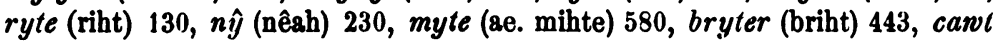
(caught) 195. - Ferner in fathyrod (fatherhood) 904, wommaned (womanhed) 1749. - In rom. worten anlautend: orebyll nach 962, abyte 1992; ebenso enabyle 683, onoryd 2022, onorabyll 950, osanna (hosianna) 2014, ower (hour) 1659, umbylnesse 2072. - exsortacion 201, enirylawns (inheritance) 2075. - erylage 1950.

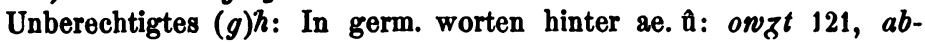
owth 206, abowzth 154 etc.; hinter ae. î: writh 1365, smyth 733; hinter ae. ê: sweth 1221. - In rom. worten: doth (doute) 42, dowth 696, stowth (afrz. estout) 373, delith 337, profyth (prophet) 581 (vgl. den vorhin in der verbalflexion erwähnten fall der schreibung von $t$ für th; $\nabla g l$ auch thunc 1642). - Unberechtigtes h anlautend in rom. worten: havdyence 460,

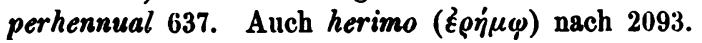

Fiir ht begegnet th: hith 1822, lyth 1594, dyth 1578, knyth 348, brygth 68 , syth 69 , ryth 126 , nyth 139 , mithy 257 , wyth 225 , wygth 227 , cawth 191, tawth 1259, browth 279 , wrowth 305 , sowth 307 , bowth 589, nowth 591 . 
Ae. hw wird verschieden widergegeben: wy 1057. 1061, wo 1062, wos 9, quat 240. 445. 523 ete., quy 1819.

Vor $n$ findet sich oft o fir $\mathrm{u}$; so im präfix on- für un-, auch ondyr 117. 266, hondyrd 653 .

Ae. sc erleidet auch metathesis und wird $\times$ geschrieben: $x$ all 28. 31). 41 etc., xold 366, xuld 1163. 2036.

In den worten, in denen ae. $d$ in ne. th iibergieng, finden wir schon regelmässig th: father, mother, thethir, hethir, wethir, togethir; so sprach schon der dichter; vgl. mother 78 (: brothyr). fader nach 48 ist wol so gut ein versehen wie odir 415, obgleich sich ja auch sonst in hss. diese form findet.

Es ist schon darauf aufmerksam gemacht worden, dass dieses stlick auch in bezug auf die metrik unbedeutend ist; die lïnge der verse, die zahl der hebungen in ihnen wechselt in unangenehmster mannigfaltigkeit, wenn wir auch im allgemeinen das bestreben des dichters erkennen können, den ernsten, gewichtigen scenen lange, gewichtige verse, den lustigen kurze zu geben. Auch der bau der verse ist hier noch holperiger als sonst und der strophenbau verderbt. Noch weniger hier, als etwa in den anderen stiucken, glaubte ich daher das recht zu haben, metrisch regelmässige verse auf kosten des überlieferten textes herzustellen; man würde meiner meinung nach sich dadurch vom originaltexte entfernen, nicht etwa ihn widerherstellen.

Auch hier sind natïrlich im auftakt keine bestimmten regeln gewahrt; für gewöhnlich ist er im anfang, wie hinter der ciisur, einsilbig, aber auch zweisilbig (1.191) oder fehlend (13.15), und ebenso nach der ciisur (1. 17.26; 10.53).

Das erstrebte und wol auch am häufigsten begegnende maass war der. heroische vers, mit der cäsur zwischen der zweiten und dritten hebung; aber auch nach der dritten hebung kommt sie vor (11.48), ebenso nach der ersten (5. 23). Sechs hebungen sind gar nicht selten, die dann die cäsur gewöhnlich nach der dritten hebung haben (13.338.422), seltener nach der zweiten (4. 27. 1934? oder siebenhebig?), sieben hebungen, cïsur nach der dritten (80.12\%,1149) oder vierten (157) oder zweiten und funften (142). Selbst acht hebungen, cïsur nach der dritten (42) oder fünften (193j?). Auch weniger als fünf hebungen kommen vor; so sind ganz gewöhnlich viermal gehobene verse, cäsur nach der zweiten hebung (z. b. 119) oder ersten (108. 611. 643) oder cäsurlos (28. 124). Bei drei hebungen steht der einschnitt nach der ersten (228.645) oder zweiten (373), oder sie entbehren desselben (41. 109. 138). Zwei hebungen haben wir in den versen 177. 945. 1481 und eine in 399. 1069.

Die grosse verwirning im strophenbau darf man nicht ganz auf die rechnung des dichters setzen; wir werden sehen, dass dieser ganze abschnitte mit deutlich erkennbarem und durchgeführtem strophenbau zu stande gebracht hat, und wenn wir danit die regellosigkeit der ersten zeilen vergleichen, wo, wenn irgendwo, doch sicher regelmässigkeit am platze war, so scheint, dass durch häufige interpolationen und sonstige veränderungen, auch nachlïssige überlieferung manches erst beim ab- 
schreiben gestindigt worden ist. Und in dieser vermutung wird man bestärkt, wenn man sieht, wie die rede des 'Imperator', z. 124 ff., nicht zu ende gefihrt ist, dass in der darstellung der erscheinung Jesu am grabe und öfter sicher die nơtigen bühnenweisungen fehlen etc. So hätte man in einer kritischen ausgabe die ersten strophen vielleicht so zu restituieren: vv. 1-2 - die auch der alliteration entbehren! - fallen weg, 3-9 bieten fragmentarisch das oft gebrauchte schema $a b a b b c b c$, es wäre also nach v. 9 ein vers ausgefallen; dasselbe schema ist rein bewahrt im zweiten abschnitt v. 10-17; v. 18-23 wäre zu streichen, auch sein inhalt ist mindestens ïberflïssig und der so erzielte anschluss sehr passend; v. 24-32 brächten wir auf dieselbe form, wenn wir nur den jetzigen v. 29 zur zweiton hälfte von 27 machen, der sich da gut anschliesst, aus der jetzigen zweiten hälfte von $\nabla .27$ den neuen $\nabla .28$ machen, dann folgte der jetzige v. 28, v. 30 fiele weg und 31-32 schlössen sich an. Ob man aber, abgesehen von der. willkuir, die bei einem solchen verfahren herrscht, das ganze gedicht restituieren könnte, ist sehr fraglich. Eher-möchte es erlaubt sein, dadurch etwas fiir die metrische reinheit zu tun, dass man die grosse zahl gänzlich reimloser zeilen aussscheidet, zunächst wo es sich ohne jede störung für den zusammenhang, ja zum nutzen desselben, nicht selten tun lïsst, so z. b. 1 (natürlich auch 2, beide alliterationslos!) 100. 145.152 (auch syntaktisch sehr verdïchtig) 310. 325 (und '329) 346 (dessen sinn auch in verbindung mit dem folgenden unklar ist) 371. 435. 437. 439.501. 529. 539. 543. 546. 630. 699 (dann auch 700) 739. 793. 916. 960. 1006 (wo dann der anschluss passender wird) 1137. 1138. 1149 (1150 schlösse sich passend an 1148 an; dabei milsste natïlich auch 1152/3 fallen, wol auch 1154, da von 1155 neuer strophenbau beginnt - jedenfalls nicht zum schaden des gedichtes) 1309/10. 1355. 1385. 1435 (höchst wahrscheinlich!) 1476. 1481 (vgl. Furnivall's note!) 1525 (dann auch 1526) 166 (und 1668, aus dem folgenden anticipiert) 1685. 2001 (so wird beim anschluss von 2002 an 2000 der sinn des ersteren verses auch klarer) 2084 (dann anch 2085).

Auch mit assonanz hat sich der dichter begnilgt, wenn ihm der reim nicht gelang. Deutlich ist das an stellen wie $136: 8$. 190:2. $950: 2$. $1174: 5:$ 6. $1241: 3$. $1406: 8$. Auch sonst noch häufig, z. b. $8: 9$ (?). $84: 5$. $143: 4$. $228: 9$ (?). $248: 9$ (?). $431: 2$. $433: 4$. $436: 8$. $460: 1$. $475: 6$ (?). $477: 8$. 489 : 90. 532 : 3. $612: 3.802: 4.803: 5.897: 9.917: 9.956: 9.961: 3.962: 4$. $1794: 6.1795: 7.1800: 1.2002: 3.2081: 2$.

Fassen wir nun die strophen ins auge, so zeigt sich, dass der dichter unter zugrundelegen verschiedener arten versucht hat, sich durch diese schwierigkeit hindurchzuwinden, dass er aber auch kein bedenken getragen hat, von beabsichtigten strophen zeilen wegzulassen, wenn ihm der reim fehlte, oder an bestimmte strophische ganze nicht dazugehörige zeilen anzufigen, wenn sich ihm bequemer reim bot. Ein solcher grundtypus ist die achtzeilige strophe mit der reimstellung ababbcbc, wie sie schon im ersten stiucke begegnete; sie kommt sechzigmal vor (z. b. 209-24. 1482-97. 1900-15). Zweimal durchgefuhrt mit aufnahme des letzten reimes der ersten strophe an erster stelle der folgenden 1253-68; ebenso mit angehängter halber schweifreimstrophe, deren letzte zeile durch reim gebunden: 1285-1308. Zweimal durchgefuhrt mit aufnahme des letzten 
reimes an zweiter stelle und angehängter vierzeiliger strophe in kreuzreimstellung, mit der vorhergehenden durch reim verbunden: 2002--31.

Folgende unregelmässigkeiten mögen hier verzeichnet werden, um den oben ausgesprochenen vorwurt $\mathrm{zu}$ rechtfertigen. Die letzte zeile des obigen schemas wird widerholt 20i4-82, oder die vorletzte 422-30. Eine zeile wird vorgeschlagen mit der ersten reimend 357-64, nur assonierend 84-92. 228-31. 249-56, falls man nicht vorzieht, diese vorgeschlagenen zeilen als reimlos zu betrachten. Auch ein vorschlag von drei zeilen mit der reimstellung $a b a \mid$ acaccdcd 2083-93. Kreuzweis reimende vierzeilige strophen werden angehängt mit der reimstellung ababbcbc;cdcd 197-208. 2054-65. 158 -98. 164i-js, oder mit umgekehrter reimstellung der angehängten zeile $7 i 6-8 i$. Weiter finden sich angehängt an den stamm ababbcbc: cdeeec 10-23, cdcddc 2032-45, cdcd|ddedde 1535-52, dccbcbbebe 1659-76. Ferner findet sich : $a b a b b c b b|d b d b b e b e| b f b f b f 305-26$ und $a b a b b c b c c \mid c d c d d c c c d 5 i-i 4$.

Eine verfeinerung dieses typus findet sich durch aufnahme der ersten reimzeile auch in die zweite hälfte der strophe $a b a b b a b a$ (zugleich weitere beispiele fir Schipper, Altengl. Metrik § 173): 49-56. 277-84. 452-9. 547-54. 564-71. 588-95. 768-75. 794-801. 869-76. 979-86. 1311-18. 1619-26. 1973-S0. Diese form findet sich auch mit angehängter vierzeiliger strophe in kreuzreim, welche den reim aufnimmt: 748-59. 776-87. 265-76. Die vereinigung beider schemata liegt vor 572-87; ababbaba| acaccbcb und in 1454-6): ababbaba | cacaadad. Ferner zu erwähnen ist 1490-1594 ababbaba | aaaacac, 887-96 aa | ababbaba, 596-609 ababba | acaccaca.

Von beiden hauptarten sind auch die beiden letzten zeilen weggefallen, sodass wir bekommen $a b a b b c$ 665-70. 788-93. 1001-6. 1932-7. 2040 -5 und ababba 285-90. Abweichungen von diesen arten: $a b a b b c b$ 33-9. 671-7. $1888-94$, $a b a b b c b b$ 1828-35, ababbcc 93-9, ababbcacc 101-9, ababcbc $c b c b$ 619-29, ababbc $|c d c d|$ ededfdfdf 631-48, a $|a b a b b c|$ bdbd 910-20.

Eine zweile hauptsächliche art des strophenbaues haben wir in den beliebten vierzeiligen strophen mit kreuzstellung (auch in Chester Plays häufig); sie begegnet vienundfunfzigmal (z. b. 921-8. 1323-30. 1705-16). Um eine zeile vermehrt ababa $131-5$, sechszeilig ababab 1792-7, siebenzeilig 291-7, achtzeilig $709-16$. 1057-64. 1962-9, elfzeilig 897-907.

In derselben weise, wie oben gezeigt ist, finden wir auch hier abweichungen, die aufzufuhren iuberflissig ist, da die behauptung uber die unregelmässigkeit des strophenbaues wol schon genugsam belegt ist; erwähnt werden mag nur die häufig vorkommende form $a b a b b$; sie begegnet eltmal (z. b. 1065-9. 1948-52).

Schweifreimstrophe haben wir 1806-11 und in der erweiterten form aabccbddb 1717-25. 1734-42. Die laiform zeigen die strophen 123-40. 462-9. 729-36. 740-7. 1155-70. 1872-87. 1916-23. Dieselbe form mit aufnahme des ersten reimes der ersten hälfte auch in die zweite hälfte: aaab aaab 1210-7. 1836-43. Hierher gehört auch die aus Dunbar bekannte fünfzeilige strophe aabab 239-43. 447-51. 700-4. 1344-8. 1401-5. $1875-9$; auch vorkommend in der form aabba $610-4$. 
Umschliessende reime mit zwei mittleren zeilen haben wir 1746-9. 1860-3, mit drei mittelzeilen 389-93. 945-9. 1787-91. 1867-71.1957-61. 2136-40, mit angehängter reimender zeile: abbbaa 987-92.

Reimpare kommen zwanzigmal vor, z. b. 40. 1086. 1970. Drei einreimige zeilen begegnen elfmal, z. b. 42.1198 .1864 . Vier einreimige zeilen 477. 523. 1798. Anch die 'lectio' in kauderwälsch ist einreimig 1186-97.

Auch von allen diesen arten finden sich weniger bemerkenswerte zusammensetzungen und abweichungen; ich habe auf diese weise in unserem gedicht circa hundert verschiedene arten des strophenbaues gezählt.

Dass unter solchen umständen eine untersuchung über reinheit des reimes eine heikle und in ihren resultaten ganz ungewisse aufgabe ist, braucht wol kaum hervorgehoben zu werden. $\mathrm{Da}$ aber ferner in einem an willkürlichkeiten und schwächen so reichen produkt eine derartige untersuchung wertlos ist, so ist sie mir wol billig erlassen. Ebenso ist es natïrlich eine missliche sache, ausmachen zu wollen, wie weit wir die ungenauen reime zu restituiercn haben, und wenn wir auch in einigen fallen kaum zögern werden, dies zu tun (z. b. $1121 \mathrm{zu}$ lesen thore: before; 736 wreke : breke $732 ; 469$ wrake : betake 465 - nicht, wie Furnivall glaubt, beteche : leche 461 -; sentens : presens 1315), so möchten wir uns doch in anderen fällen, wo der reim leidet (vgl. vorher), kaum dazu berechtigt fuhlen.

\section{Moralität Wisdom.}

Das letzte sttick der hs. ist eine moralität, gehörig zu der sammlung, welche nach dem einstigen besitzer der hss., denen sie angehörten, Macro Moralities genannt werden; die Macro-hss., jetzt Mr. Gurney gehørig, sind noch nicht ediert. Die moralität, von der ein bruchsttick sich in der Digby-hs. findet, ist nach Collier, H. E. D. P., 1831, II, 87 note - der auch eine vollständige inhaltsangabe gibt - von derselben hand geschrieben wie das volltändige sttuck in der Macro-hs. Collier betitelt es nach den passiven helden: 'Mind, Will, and Understanding', Furnivall nach dem aktiven helden: 'Wisdom'. Es schildert, wie Wisdom, d. h. die zweite person der heiligen dreieinigkeit, Christus, sich von der Anima auf kurze zeit zurtickzieht, und Anima, nur ihren natturlichen kräften: Mind, Will, Understanding therlassen, von Lucifer verfthrt wird und fällt, sodass sie bei der katastrophe erscheint 'in the most horybull wyse, fowler than a fend', während die seelenkräfte in den ihnen entsprechenden stlnden schwelgen, bis Wisdom wider in die handlung eingreift und, sich der seele wider annehmend, sie auf den pfad der tugend zurtlckleitet. Dabei bewegt sich das sttick in seiner darstellung in dem gewöhnlichen rahmen, 
strent bibelsprtiche ein (z. b. z. 164 und die folgende anweisung gleich Cant. Cant. I, 4; zz. 169/70 gleich Cant. Cant. I, 5) und bietet also in bezug auf seinen inhalt nicht viel interessantes. Zu erwähnen wäre nur, dass es widerholt franzősische sitten rugt (vgl. v. 517 und im auszuge Collier's II, 291, bei uns s. 167) und einen scharfen tadel enthält gegen das Holborn Quest (das geschworenengericht des londoner stadtteils Holborn), dessen sechs beisitzer: Wrong, Sleight, Doublenesse, Falsehed, Ravyne, Diceyte sind. Es könnte das einen anhalt zur datierung des stluckes geben, wenn etwas entsprechendes tuber diesen inquest bekannt wäre.

Die erwähnung dieser londoner einrichtung wie auch dortiger lokalitäten (Westminster, St. Paul Cathedral) liessen vermuten, das sttick sei für und in, wenigstens in der nähe von, London geschrieben, womit freilich im widerspruche steht die sprache, die der grenze zwischen dem westlichen mittellande und dem norden angehört. Wahrscheinlich sind daher diese lokalen anspielungen erst später, nachdem das sttick nach stden gekommen war, woher unsere abschrift stammt, eingeftigt worden.

Jene grenze aber als heimat unseres dialektes anzunehmen, zwingt uns der umstand, dass wir sowol das hauptcharakteristikum des mittellandes, die endung - $n$ im plur. praes. ind, wie auch das des nordlandes: die endung $-\mathrm{s}$ in derselben form fir den dichter durch den reim gesichert vorfinden. Z. 56 begegnet bene (: clene 54); so anch innerhalb des verses 257. 364. 576 etc; fallyn 438; dagegen telles 270 (: dwelles, sing.); dredys 634 (: spede is, oder ist dredys sing.? das kollektiv folke ist subjekt); rechases 724 (: grace is). Wollten wir nicht fur die heimat des stlickes einen grenzdistrikt, wo die verschiedenen formen zusammentrafen, halten, so mlissten interpolationen vorliegen, die anzunehmen wir doch hier kaum berechtigt sind. - Ausserhalb des reimes hat der stldlandische schreiber seine formen -th eingefuhrt (zz. 53. 178. 605 etc.).

Die 3. sing. ind. praes. zeigt im reime regelmässig die endung -s: has 177 (: place), 578 (: grace); mase (makes) 581 (: lace); auch dwelles 272 : compelles : spelles, da sie sich im reime rein erhalten haben, wie die umherstehenden fornen -th innerhalb des reimes zeigen, mügen angeftihrt sein. Sehr selten noch begegnet die form ausserhalb des reimes, z. b. trumpes nach 694. Gew 6 hnlich hat der schreiber seine formen -th eingeftihrt (zz. 5. 11. 13 etc.), flexionslos ist seme 475.

Für nördliches mittelland spricht die doppelte behandlung eines ae. â; dies findet sich gewöhnlich in der form $\overline{0}: l o 274$ (: meo), rore 325 (: restore : lore), lore 418 (: before). Dagegen auch als a: mare 742 (: hare). Innerhalb der verse begegnet regelmässig $\bar{o}$. Ebenso rïhren vom schreiber her die suidlichen aus dem Altenglischen erhaltenen formen des pron. pers. 
3. plur.: hem 3. 56. 293 etc., here 636, nach 694. 726, nach 754 - neben den gewöhnlichen nördlichen formen. - Gegen rein nördlichen dialekt - spricht der umstand, dass das part. praes. starker verba sich auch ohne -n findet: be 130 (: see), take 221 (: wake), bore 419 (: lore). Auch part. praes. -and, nach Morris eine eigentümlichkeit des nördlichen und mittellindischen dialekts, findet sich: wymnand 679 (: stand), reynand 681 (: stand), usande 683 (: land). Innerhalb der verse steht -ing.

Ae. $y$ als umlaut eines älteren $u$, 0 wird $z u$, geschrieben $y$, und reimt mit $i$, e ( $i$ und e reimen auch sonst): synne (: withinne) 158, kynge : thinge 289 etc., mynde : kynde : hende 42, knetle : dette 196 etc. Innerhalb der verse wird auch gewöhnlich y geschrieben: mynde 185. 197, kysse 596, synne 224. 235; auch $\mathfrak{a}$ in lustes 51. 460. 516, oder e in mende 55. 183.

Ae. $d$ ist noch durchgängig erhalten, wo es ne. in th ïbergeht: fader, togedir, thedir, hedir finden sich oft (whedyr, ae. hwæðer, 526). - Auch hier begegnet in einigen worten -gh- nur als längezeichen für ou, $\hat{i}$ : abought 464. 501, dought 502, lought 503, whight nach 16 (aber white 155, nach 164), perfight 233. 699, dispight 338 .

Im versbau begegnen dieselben erscheinungen wie auch sonst; gewöhnlich einsilbiger auftakt, auch nach der cäsur, aber auch zweisilbig (3. 4) oder fehlend (14. 25. 41); nach der cäsur selten zweisilbig (2. 246. 254) oder fehlend (3.4). - Die hebungen in den versen wechseln zwischen zwei und sieben; wol die mehrzahl der verse hat vier hebungen, ciisur (oft durch punkt bezeichnet) gewöhnlich nach der zweiten hebung (18. 40. 42. 132. 133), auch nach der ersten (30. 34) oder cäsurlos (1. 6. 32); bei fünf hebungen steht die cäsur nach der zweiten (4. 25. 39. 141) oder dritten (5. 11. 14. 41. 57) oder ersten (155. 281). Bei sechs hebungen nach der dritten (68. 252. 483) oder vierten (280. 283), selten zweiten (143). Bei sieben hebungen nach der dritten (13. 37. 483), vierten (7. 89. 702), selbst zweiten (8). Ziemlich häufig sind auch drei hebungen (149. 328-31. 333-6. 353 etc.). Zwei hebungen haben $325-i$. 352. 392. 513. 424.518 etc.

Die reime sind im allgemeinen rein, besonders in bezug auf die konsonanten; gewöhnlich liegt stumpfer reim vor, nicht selten klingender, z. b. 253 desiderable : delitable; 270 telles : dwelles : compelles : spelles; 634 dredys : spede is; 719 traces : faces : space is : places : rechases : grace is; 730 togedyr : thedyr etc. Auch gleiche reime sind nicht selten, z. b. $34: 36,218: 20,486: 8,663: 4: 5$. - Gegen assonanz verstossen: favour : fygure 209; try (true) : lye : affye 643; whyppe : up 518; gesse : is : mys 401; yiftes : thrift is 642. - Gegen konsonanz verstossen: wykednesse : perverse 378. $732: 3$ bait : endyrect (oder indreit? zu droit; das subst. droit ist ja noch ne. dial). - $\mathrm{Zu}$ emendieren sind: 206 creatour, 402 this, 497 agre $I(?), 525$ roume; 58 in especyall; 297. 530 kende (? vgl. mankende 44); 189.528 mende(?) 590 ware; 682 whore.

Im strophenbau haben wir zwei hauptarten achtzeiliger strophen: die schon widerholt vorgekommenn mit der reimstellung ababbcbc $(1-24$, 221-76, 293-324 etc.); zusammensetzungen mit dieser form ababbcbc+ cacaadad 20-40, + cdcddede | efeffgfg $77-100,+c d c d d e d e \mid$ fefeegeg $\mid$ ehehhihi 125-56, +dcdccece 173-88 (dabei sind die drei zeilen zwischen 
179 und 181 zu einer zu vereinigen); +dbdbbebe $205-20,177-92 ;+c d c d$ (e ?) 49-61). - Die zweite form ist die des lay - auch in den kollektivmysterien sehr oft gebraucht - mit der verzierung, dass der hauptreim der ersten hälfte auch in der zweiten widerkehrt, z. b. 325-452, 583-662 etc. Auch 492-517 gehört hierher, nur muss man - in der zählung der verse ist es übersehen, vgl. dagegen z. 181) - 510-11 zu einer zeile vereinigen. Zusammensetzungen mit dieser form: aaabaaab | bbbcbbbc 453-68. Noch künstlicher aaabaaab | aaacaaac 663-78. Abweichungen davon aabaaab 552-9, aaabaub 576-82 (hier hätte Furnivall konsequenter weise auch raum lassen sollen für eine einzuschiebende zeile zwischen 579/82, wie zeile 553, oder es in beiden fällen auf der überlieferung beruhen lassen). Vereinigung beider hauptarten haben wir in aaabaaab | cbcbbdbd 477-92. Reimpare zum abschluss 5/8. 735 . 745.

\section{The Burial and Resurrection of Christ.}

Das stick, welches Furnivall seiner ausgabe noch beiftigte, ist furr uns von grösstem interesse, da es das einzige mittelenglische dramatische produkt ist, in dem die lyrischen teile einen so grossen platz einnehmen. Schon in den kollektivmysterien finden sich bei der darstellung der kreuzigung derartige lyrische stellen, aber immer nur als nebensache und kurz. Bei uns bilden sie gerade die hauptsache, sodass die dramatische handlung daneben ganz zurticktritt, die in jenen sammlungen ganz im vordergrunde steht. So nähert sich dieses sttlck sehr den deutschen religiösen dramen. Unser dichter hat aber auch gar kein drama geschrieben: er verfasste einen 'treyte or meditatione'; demgemäss setzte er denn auch die einleitenden worte und die anweisungen, z. b. wer spreche, regelmässig hinzu. ${ }^{1}$ Erst der schreiber, der sich vom dichter durch einen abweichenden dialekt unterscheidet, kam, und zwar auch erst als er etwa bis v. 81 gekommen war, auf den gedanken, das gedicht in ein drama $z u$ verwandeln, und so strich er denn die jetzt uberflussig gewordenen anweisungen zum sprechen etc. und setzte, da am anfange kein platz mehr war, auf den rand der rickseite von fol. 140 die anzeige: 'This is a play etc.', die Furnivall dem stticke vorgesetzt hat.2 Und so, der nenen behandlungsweise eingedenk, liess der schreiber die einfuhrenden

1 Vgl. vv. 14. 15, note zu 55 etc.

2. Aus dem eben dargelegten erhellt, dass der herausgeber dem dichter unrecht tut, wenn er ihm jene scheinbare unaufmerksamkeit zur last legt und ihn 'poetasler' nennt (s. 174 note 2 u. ö.). Gerade der dichter des vorliegenden stiickes verdient dies prïdikat am allerwenigsten. 
worte weg bis v. 138. Da aber verliess ihn die nötige aufmerksamkeit, und nun wechselt blosses abschreiben mit umarbeiten bis zur anweisung vor v. 419; dann hatte er sich durchgearbeitet und behandelte das folgende fehlerlos als drama. Dabei kam ihm sehr zu statten, dass, wenn er die anzeigen der einfthrung einer person, die ja jetzt uberfltussig warden, wegliess, er den strophenbau deswegen nicht zu zerstören brauchte, da der dichter schon die verse, die als anweisung dienten, nicht in der strophe zählte, obgleich er sie dem reime einpasste.1 Dabei fehlen auch jetzt noch immer die sonst so gewöhnlichen buhnenweisungen fast ganz.

Regelmässiger in jeder beziehung ist der zweite teil, der wol von demselben verfasser und abschreiber herrïhrt, wie der erste (vgl. Forewords s. VII). Zu gunsten dieser annahme lässt sich anfthren, dass in der hauptsache beide dieselbe sprache und orthographie und dieselbe einzig dastehende art der behandlung zeigen, wenn das anch hier, wo reichere dramatische handlung geboten war, nicht so scharf hervortritt; anch der strophenban ist ein gleicher in beiden; ihrer beider ist in der tuberschrift 'This is a play etc.' als eines ganzen gedacht (wohhrend im prologe freilich nur vom 'Burial' die rede ist) und in der hs. schliessen sich beide ohne unterbrechung an einander an; endlich scheint der schluss des ersten teiles (vgl. vv. 845 ff., 856 ff., 859, besonders 860) auf den zweiten vorauszadenten. Sind aber diese grttnde an sich schon nicht zwingend, so finden sich einige wenige, wenn auch ganz geringe unterschiede in der sprache, sodass ich die frage nicht definitiv zu entscheiden wage.

Der inhalt, so weit er nicht, was ja freilich gerade die hauptsache ist, der brust des dichters entsprang, ist entnommen der bibel und dem gottesdienst; seltener finden sich zthge aus der legende. Das sttlck ist interessant genug, insofern als es sich eng an den gottesdienst anschliesst und fast nur eine weitere ausfthrung dessen gibt, wozu der dichter durch die lyrischen elemente der liturgie: antiphonen und responsorien angeregt

1 Vgl. z. b. nach v. 61, wo wir sonst fünf reimende zeilen hätten im erweiterten schweifreim, und später, wo die anzeige des sprechenden der reinen schweifreimstrophe immer eine überflissige zeile hinzuftigte, oder die note zu $\nabla .391$, wo die erzählenden worte einen strophenbau zeigen, der sonst gar nicht vorkommt. 
wurde, ja schliesslich mit dieser verschmolz, indem es die im gottesdienste gesungenen lieder und die darin vorkommenden dramatischen handlungen in sich aufnahm. Ein blick auf den gottesdienst jener zeit zeigt uns, dass der zu bearbeitende stoff dem dichter voll in der kirchlichen feier der passionszeit geboten wurde, indem neben der lesung der evangelien besonders die sequenzen betrachtungen ther das leben und wirken Jesu anstellten, die sich denn bei uns noch weiter ausdehnen, sonst aber ganz in der art jener gehalten sind. Sogar der strophenbau, dessen sich unser dichter bedient, scheint direkt den sequenzen entnommen zu sein, die sowol in schweifreim ${ }^{1}$ wie auch in der erweiterten form des lay ${ }^{2}$ verfasst sind. Wenn wir nun am ende des zweiten teiles - nach v. 1556 - sehen, dass in das sttick aufgenommen sind die dramatischen zttge, die auch in der kirche zur darstellung gelangten (vgl, Guéranger, Année liturgique, in der ausgabe von Heinrich bd.VII), und die gesänge, die dabei vorgetragen worden, und daneben hören, dass dieser drammatischen darstellung des zusammentreffens der apostel mit Maria Magdalena nach der erscheinung Jesu vorhergieng eine prozession, welche den zweck hatte, den gang der heiligen frauen nach dem grabe darzustellen ${ }^{3}$, und ebenso eine art dramatischer widergabe des zusammentreffens des engels mit den frauen (diese schilderung geht der von Furnivall s. 227 mitgeteilten, bei Guéranger-Heinrich VII, 163 stehenden voran), so erkennen wir leicht, dass unser ganzes sttick nur eine realistische darstellang dessen ist, was in abgeschwächterer form schon in der kirche selbst geboten wurde, also zurtickkehrte in den dienst der religiossen institution, welcher das drama therhaupt sein dasein verdankt. Auch der erste teil, das begräbniss Christi, ist dann wol dem wunsche zuznschreiben - gleichgiltig ist ja hierbei die änderung der form -, da eine quasi-dramatische darstellung der erzählung der biblischen geschichte am charfreitage sich nicht im gottesdienste in der art findet, wie am ostersonntage, diese durch das gedicht zu ersetzen.

' Z. b. Sequentia der Feria sexta post Pascha bei Henderson, Missale Ebor. I, 133, anch in den Seqq. communes in commemor. beatae Mariae bei Henderson a. a. o. II, 209 etc.

2 Ib. II, 206. 208 etc.

3 Guéranger, Année liturgique, ed. Heinrich VII, 58. 
Der erste teil wird eroffnet durch Joseph von Arimathaea, dem sich die drei Marien zugesellen, alle jammernd uber Jesu tod and sich der einzelnen, aus der leidensgeschichte wolbekannten scenen erinnernd. Wir erfahren aus ihren worten, dass sie sich am fusse des kreuzes befinden, an dem Jesns noch hängt. Magdalena schildert die seelenqualen der jungfrau Maria, besonders als ihr sohn zu allen, nur nicht zu ihr sprach (dieser zúg ist in der mittelalterlichen darstellung gewöhnlich; vgl. z. b. Coventry Myst., Cracifixion, s. 322, Maria: Thou hast spoke to alle tho that ben here And not a word thou spekyst to me!). Er habe sie darauf seinem vetter Johannes empfohlen (Joh. XIX, 26/7). ${ }^{1}$ Sie erinnern sich seiner worte: 'I am thrustye' (Joh. XIX, 28: Sitio), die aber zu beziehen seien auf seinen durst nach dem heile der menschheit. ${ }^{2}$ Wenn in v. $271 \mathrm{ff}$. Jesu körper einem buche verglichen wird, dass so viele blutige buchstaben enthalte, dass wenig rand tibrig geblieben sei, und einem auseinander gezerrten pergament, so verrät nur das den gelehrten verfasser. Der einzige trost der trauernden ist die erinnerung an sein versprechen, wideraufzuerstehen am dritten tage (Matth. XVI, 21 u. o.). Ihr jammer mttsse gross sein, da sogar. die unbeseelte schöpfung aus ihren gewohnten bahnen gewichen sei: die sonne (Mare. XV, 33; Luc. XXIII, 44/5), wie anch die erde und felsen (Matth. XXVII, 52). Dann kommt Nicodemus zu den trauernden; er zurnt den juden, dass sie den gott gekreuzigt haben, der sie einst in der wilste vierzig jahre lang leitete, mit engelbrot speiste und in das land der verheissung fthrte. ${ }^{3}$ Er erinnert sich der unterredung, die er einst mit Jesu hatte (Joh. III), und schliesst mit dessen spruche: Nemo ascendit in celum nisi qui descendit de celo

1 Ueber die verwantschaft belehrt uns Leg. aur., De nativitate beatae Mariae virginis: mortuo autem viro Annae [mutter der jungfrau Maria] tertium accepit, scil. Salome, ex quo aliam filiam genuit, quam rursus Mariam vocavit et Zebedaeo in conjugem tradidit. Haec autem Maria ex Zebedaco, viro suo, duos filios genuit, scil. Jacobum majorem et Jo- hannem evangelistam.

\& Vgl. Missa de V vulneribus Jesu Christi, Sequentia, bei Henderson, Missale Ebor. I, 254: loquens Jesu postea, Sitio, dicebat... Num quid hanc [scil. crucem] doloribus magis sentiebas, Sed salutem potius nostrum siliebas? Ein allgemein verbreiteter zug, auch in deutschen sticken, z. b. Sterzinger Passion: Sitio! Mich dürst nach dem heil der menschheil.

3 Vl. Improperion des charfreitags, Guéranger-Heinrich VI, 542: quia eduxi te de terra Aegypti... quia eduxi te per desertum quadraginta annis: et manna cibavi tibi, et introduxi te in terram satis bonam optimain, Missale Ebor. I, 105. 
(Joh. III, 13). Als sie sodann Jesum eben abgenommen haben vom kreuze, kommt die jungfrau Maria dazu, den tod ihres sohnes bejammernd, sie fällt in ohnmacht. ${ }^{1}$ Sie erinnert sich der vergangenheit: der mission Gabriel's an sie (Luc. I, $26 \mathrm{ff}$.), seiner verheissung der freude und im gegensatz dazu der prophezeiung Simeon's (Luc. II, 35), die jetzt in erftllung gegangen sei: jetzt habe das verheissene schwert ihre brust durchbohrt ein ganz gewöhnlicher zug. ${ }^{2}$ Schon frtiher wurde darauf hingewiesen, dass diese rilhrenden klagen und bitten Maria's das schönste sind, was wir in diesen spielen finden und wirklich nicht ohne wert, auch fir uns: sie sind einem warm fuhlenden herzen entquollen. Sie halten sich in dem gewöhnlichen rahmen religiöser lyrik, mit den in solchen dichtungen besonders häufig gebrauchten achtzeiligen strophen mit der reimordnung ababbcbc oder den auch sonst vorkommenden rhymes royal; eine bestimmte ähnlichkeit, die auf eine quelle deutete, findet sich nicht: quelle war auch hier des dichters inneres. ${ }^{3}$ Einzelne zuge begegnen immer wider, so die anklage der juden, die bitte an den grausamen tod, auch sie hinwegzunehmen; ganz besonders beliebt war das bild des kindes Jesus an der mutterbrust, und die hinweise darauf sind unzählig in der mittelenglischen religiösen lyrik uberhaupt, wie auch bei uns 4; in ihrer hauptklage wendet sie sich namentlich an Magdalena, Cleophe (Maria Cleophae Joh. XIX, 25) und Jakobye (Maria Jakobi, Mare. XVI, 1). In dieser klage begegnet widerholt der refrain: Who can not wepe, com lern at me, auch in anderer ähnlicher form, der entnummen ist einem älteren hymnus, publiciert von Furnivall (Hymns to the Virgin and Christ, E. E. T.S., 1867, s. 126), mit dem unser abschnitt aber sonst keine nähere ähnlichkeit zeigt; auch der

1 Ebenso in Coventry Pl., Crucifixion, deren darstellung überhaupt der unseren am nächsten kommt; ähnlich die darstellung im Burial of Christ in jener sammlung.

2 Vgl. z. b. Kindheit Jesu, ed. Horstmann, 1875, v. 919: this swerd was oure lordes deth that thorn hire herte wende under the rode.

$3 \mathrm{Vgl}$. besonders The Lamentation of the Virgin, auch in nordlicher sprache, auch mit dcm strophenbau $a b a b b c b c$, auch mit refrain, in Reliquiae Antiquae ed. Wright und Furnivall II, 213; oder Furnivall's verschiedene ausgaben solcher gedichte, besonders in den Political, Religious, and Love Poems s. 204 ff.

4 Z. b. in dem refrain: Which sumlym gafe you mylk of my pape oder Remembere myn awn der son, that ze sowket my breste; vgl. dazu das von Wright, Chester Pl. II, $204 \mathrm{ff}$. mitgeteilte gedicht mit dem refrain: The chylde is dedd that soke my breste. 
strophenbau in beiden ist verschieden. Entlehnt ist auch das 'Speciosus forma' v. 645.'

Nach dem begräbniss endigt der erste teil mit der ausgesprochenen absicht der Magdalena, Jesu körper einzubalsamieren, und der hoffnung Joseph's, Jesu werde auferstehen, und die zeit nicht mehr fern sein, wo er diese freudige nachricht hören werde. Da setzt der zweite teil ein: die drei Marien auf dem wege zum grabe, um den leichnam Jesu zu balsamieren, sein schicksal bejammernd und doch voller hoffnung, denn der verheissene dritte tag sei ja nun da. Der engel erscheint (Matth. XXVIII, 2; Marc. XVI, 15), fragt, wen sie suchen. Sie antworten: den gekreuzigten Jesus von Nazareth (nach Marc. XVI, 6; Matth. XXVIII, 5). Aber es wird ihnen zur antwort, er sei nicht da, er sei auferstanden (Matth. XXVIII, 6), er werde seinen schulern erscheinen (Marc. XVI, 7); und als Magdalena doch noch weint, fragt er sie: Mulier, quid ploras? (Joh. XX, 13); sie antwortet, weil ihres herren leib fort sei. Er verkündet ihr die auferstehung, sie solle näher treten, wo er gelegen habe (Marc. XVI, 6), in Galilaea werde er erscheinen (Matth. XXVIII, 7). Aber Magdalena ist, wie die folgende unterredung mit den beiden Marien zeigt, noch wenig getröstet, macht sich vielmehr vorwtirfe, der auferstehung aus pflichtvergessenheit nicht beigewohnt zu haben.

In der zweiten scene tritt Petrus auf 'flens amare', und er behält diese tränenreiche rolle bei. Der zug stammt aus der legende, die ihn, und das ist ja ganz naturlich nach seiner verläugnung Christi (Matth. XXVI, 70 u. ö.), mit vorliebe beständig weinend darstellt.2 $\mathrm{Er}$ erinnert sich seiner herkunft, seines frtheren namens Symon Bariona (Leg. aur. a. a. 0.: Petrus trinomius exstitit. Vocatus est enim Symon Bariona etc. Matth.

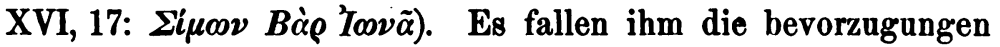
ein, die Jesus ihm vor anderen zu teil werden liess, da er ihn mit Johannes und Jakobus zur 'highe speculation' seiner 'godly maiestye' in der 'transfyguration' berief (Matth. XXVI, 37). In dieser weise fthrt er noch mehr bekannte ereignisse aus Jesu

I Vgl. Dominica infra octavas nativitatis, Gradale, bei Henderson, Missale Ebor. I, 28, II, 329: Speciosus forma prae filiis hominum.

${ }^{2}$ Vgl. Leg. aur., De So. Petro Apostolo: Quando etiam culpam negationis ad memoriam reducebat, ubertim lacrymas emitlebat, unde adeo in consuetudine habuit flere, ut ejus facies tota adusta lacrymis videretur. 
leben an, um seine schuld darzulegen. Vergebens versuchen die beiden anderen jünger ihn zu trösten. Andreas macht ihn aufmerksam auf Jesu voraussicht: 'Omnes vos scandalum patiemini' und: seine heerde werde sich zerstreuen, wenn der hirte geschlagen sei (Matth. XXVI, 31). Er erinnert ihn an Jesu grosse liebe und sein gebot: siebenundsiebenzigmal statt siebenmal zu verzeihen (Matth. XVIII, 21), seine verheissung: 'Quodcumque ligaveris ete.' und die tibertragung der schltssel fur himmel und hölle (Matth. XVI, 19). Endlich lässt er sich trösten und bricht in die bitte aus: Ostende faciem tuam et salvi erimus. ${ }^{1}$

Nachdem so, nicht ohne geschick, die erscheinung Jesu von verschiedenen seiten her vorbereitet ist, findet diese selbst statt. Magdalena, jammernd, erwartet den herrn. 'Quem diligit anima mea, quaesivi; quaesivi illum et non inveni (Cant. Cant. III, 2). Filie Jerusalem, whereos ye goo, Nunciate dilecto meo quia amore langueo. ${ }^{2}$ Da erscheint ihr Jesus in specie ortulani (nach Joh. XX, 15), fragt sie: Mulier [quid wol nur aus versehen ausgelassen] ploras? quem queris? Sie hält ihn fur den gärtner. Er ruft sie an: Maria (Joh. XX, 16), und sie antwortet: Raboni (Mare. XVI, 9); dann sein: Noli me tangere (Joh. $\mathrm{XX}, 17)$ und der auftrag, nach Galilea zu gehen und den bridern (Matth. XXVIII, 10), besonders aber Peter (Marc. XVI, 7), zu sagen, er sei auferstanden und werde ihnen erscheinen. Jetzt ist aller kummer aus Magdalena's seele, und sie jubelt den beiden anderen Marien entgegen. Darauf erscheint Jesus allen dreien mit dem rufe Avete (Matth. XXVIII, 9).

Jetzt hatte der dichter das spiel so weit gefuhrt, dass die quasi-dramatische darstellung der kirche sofort eingreifen konnte, und das geschieht denn auch in der gewöhnlichen weise (vgl. Guéranger-Heinrich VII, 163), eingeleitet durch das lied: Iictime paschali laudes immolent Christiani (s. Feria III, post Pascha, Sequentia, bei Henderson, Miss. Ebor. I, 129). Eine

' Vgl. In transfiguratione Domini, Introitus, bei Henderson, Missale Ebor. II, 212: Veni et ostende faciem tuam, Domine, qui sedes super cherubim, et salvi c'rimus. Auch bei Simmons, The Lay Folks Mass Book 1879 , s. 70,33 im York Bidding Prayer: Et ostende faciem tuam el salvi crimus; ebenso ib. 77,$28 ; 92,20$.

2 Cant. Cant. V, S. Diese stellen aus dem hohen liede sind oft verwendet; vgl. Furnivall, Hymns to the Virgin and Christ s. 2, 18; 3, 49. 57. 39. 60. Furnivall, Political, Religious, and Love Poems ss. 148. 150 zwei lieder in drei fassungen mit dem refrain: quia amore langueo, in achtzeiligen strophen mit der reimstellung $a b a b b c b c$. 
weitere ausfthrung dessen, sowie ausrichtung der anfträge Jesn schliesst sich dem noch an. Die jünger eilen zum grabe, Johannes voran (Joh. XX, 4), grosse freude herrscht anch bei ihnen, und mit einer verherrlichung Christi schliesst das sttick.

Es ist wol kaum zufall, dass unser stlick etwa der gegend angehört, seinem dialekte nach, aus der uns eine reichere religiöse literatur vorliegt. Wir haben in ihm nördliche dialekteigenttumlichkeiten gemischt mit mittelländischen, und zwar ostmittelländischen, nicht westmittelländischen, wie Morris s. 170 unserer ausgabe behauptet.

Morris und Furnivall glauben nun, es sei ursprünglich in nördlicher sprache abgefasst und erst durch den schreiber seien die mittelländischen formen hineingekommen. Das ist jedoch eine unbegrïndete annahme; im reim findet sich kaum irgend etwas, das zu dieser behauptung berechtigte. Wenn Morris s. 170 unserer ausgabe sagt: 'whole lines have been altered to get a midland ryme', so kann er auch das nicht beweisen, und das beispiel, das er als beweis gibt, ist jedenfalls nicht glïcklich gewählt. Er glaubt nämlich, dass die form sho (für she) in unserem denkmal die ursprüngliche sei, die nur durch den schreiber oft verderbt sei, und will diese nun auch in $\nabla .204$ einfuhren: 'what wordes saido sho', und glaubt daher, $\nabla .203$ sei geändert aus 'she was full wo'. Dann ist aber wider wo keine nordliche form und ferner findet sich she auch sonst noch reimend, z. b. v. 918, und endlich gehört in v. 204 tiberhaupt nicht she, sondern he, denn der gegenstand der unterhaltung wechselt, es wird nicht mehr von Maria, sondern von Jesu gesprochen, wie die folgenden zeilen unzweifelhaft beweisen. Ungerechtfertigt ist es natürlich auch, wenn er für v. 918/9 vorschlïgt: 'that sho ... wald with me goo', denn go so gut wie wo ist nicht nördlich.

Um nun auf die formen selbst einzugehen, so finden sich an nördlichen eigentilmlichkeiten: plur. praes. ind. -s: sais nach $\mathrm{v} .55$, hase 161, spekes 163, flowes 371, rynnes 644 etc. So auch is 683 (vgl. Zupitza zu Guy of Warwick 3194). - Dazu stimmt 3. sing. ind. praes. -s: hinges 71. 116. 232. 233 (i aus praet. hing? 176 neben hang 677; auch der inf. zeigt i: note zu 391. 397. 308; auch im intrans. 762), sloes 73, passis 134, haves 136. 282. 286, hase 289, fayntes 359, approchis 859. - Auch 2. sing. ind. praes. -s: is 383 , haves 403 . - Part. praet. st. vb. hat gewöhnlich -n, doch throng (: son) 670. - Part. praes. -ing.

Ae. â findet sich erhalten in chase, ae. cêas, 530; vgl. anch knawen 5. 496. 653; lawe, an. lâgr, 2; awn 183. 401. 730 u. ठ̌. - till vor inf. ist sehr häufig: 428. 528. 608. 846 etc.; neben to 594. 595. 596. 626 etc. Auch sonst ist till häufig für to: till a stokke 402, till a tree 673, till Egypte 777, till us 799 etc.; intill für into: intill egiple 633. - Ae. y, umlaut eines älteren $u$, o hat regelmässig $i$ ergeben (geschrieben $y, i)$, auch für den dichter gesichert: mynde 42 (: fynde), syn 295 (: in). In thrusten 214. 220, ae. thyrstan, ist einwirkung des subst. thruste 215 , ae. thurst, und des adj. 
thrustye 210, ae. thurstig, zu erkennen. Durch vorherrschen des $i$ sind auch zu erklïren formen wie mich 132. 335, brisle 780, hir 179, sich 299. 783. 34, neben such 300, ristc 779 , ae. restan. - Als spezifisch nördliche wörter sind wol nur yrk (schwed. yrka) 111 und ilk (schott. ilk) $844 \mathrm{zu}$ nennen. Hier mag auch erwähnt werden awe 4. 7.653 für und neben all 104. 419. 585 etc.; vgl. Halliwell, Dict. of Archaic and Provincial Words 1847, I, 120: 'an = all.' North.; Jamieson, Scottish Dict. I, 84: 'aw = all'. Vgl. auch noch wald später.

An mittelländischen eigentiimlichkeiten findet sich plur. praes. ind. -n in ben 193, beyn 272. 290. 355. - Fiir den osten spricht 3. sing. praes. ind. -th: callith 244, hingeth 337 , doth 613.811.820, drawethe 859 .

Wenn sich ae. â als $\bar{o}$ findet, durch den reim für den dichter gesichert, so ist damit noch kein sicheres merkmal zur lokalisierung gewonnen, da ja $\delta$ im laufe der zeit auch in den norden, wenn auch nur vereinzelt eindrang; so findet sich beholde 366 (: golde), 546 (: molde), 697 (: wold); loo 748 (: doo); goo 751; goo (: two) note zu 55.

Für die späte zeit der abfassung zeugt, dass der dichter in den worten, in welchen ae. $d$ später in th übergieng, schon th sprach. So findet sich mother 170 : other; derselbe reim, trotzdem da moder geschrieben ist, steht $134: 8$. Ausserhalb des reimes begegnet mother noch viermal. Ebenso steht hither 128. 271. Die form moder dreinnddreissigmal; ebenso regelmässig fuder, togider, whider. - Ebenso ist ein zeugniss für die späte zeit der abfassung der auch in den norden eingedrungene ubergang von $e$ zu $a$ vor $r$, z. b. hart 836 (: depart); dieselbe erscheinung auch in warkes 34 ; und das verstummen des $g h$ nach $i, z . b$. righte: contrighte 252.

Erwähnenswert scheint noch folgendes zu sein: Fïr $\hat{o}$ ist oft $\mathbf{u}$ geschrieben: gud 6. 14. 25 (: floode), aber good 93; blude 286, aber blood 177; rude 604. 677, aber rood 129; fute 685; buke 272; luk(yd) 189. 696; suthe 500; mude note zII 391. - Neben she 203 (: hee), 179 kommt sho vor z. b. 159. 172. 177. - Neben as 49. 191 etc. kommt os vor 35. 156. 225 etc. - Ae. $\mathrm{cw}$ ist durch wh widergegeben in whik(lye) 444. 814. Ebenso frz. qu in whantile 621.737, whit 850. - ey tritt ein für 1. 2e. $\hat{\mathrm{e}}$ in ceile, se. sêl, nordh. sêl, 72; leyf 466. 2. se. ê in keyle, se. cêlan, 76; feyle 97. 3. ae. $\mathrm{e}$ in weyll 79. 84 neben well $390 \mathrm{u}$. 8 . - wald, nordh. walde, findet sich 88.126.153 etc. für und neben wold 401. 559. - Praet. und part. praet. schw. verba zeigt -t: cessit 8 , wepil 15, levil 16, blessit 30) etc.; aber auch -d: lakid 122, verryfyed 43, changid 110. - th fir t findet sich in comfurth 261.419.454 u. ö. - Schwache konjugation zeigt taken im part. praet. takid; ebeso beten in derselben form bett 684; vgl. Havelok 1916 bei Stratmann, Dict.; Zupitza zu Guy 294!, auch zu 5367. Neben lambe 238. 254 etc. findet sich auch $\operatorname{lam}(e)$ 206. 223. 224 etc.

Auch der zweite teil bietet im allgemeinen dieselben sprachlichen crscheinungen wie der erste. Plur. praes. ind. -s: has 887. 1305. 1426; sais nach 1001. Oder $-\mathrm{n}$ : beyn 132\$. 1548. - 3. sing. praes. ind. -s, auch für den den dichter belegt: fayles 1398 (: nayles), lyes 931, gyves 95!, commes 970. 1194, faris 1095, haves 1543. 1552, has 1332. 1323. Dagegen findet sich -th: doth 590 . 1051, lyeth 980. - 2. sing. ind. praes. -8: wepis 
1007. 1469, sekes 1469. - till vor inf. 992. 1252. 1315. 1335. 1345. 1580. Auch sonst oft für to: till a tree 878, till his payn 949, till us 1061. Ae. â erhalten: maste (mâst zu mâst) : paste 953, wât 1010, are (ae. âr) 1188, chace (ae. cêas) 1306; vgl. knaw 937. 1141. 1329 etc., awn 883 (own 1149), lawly 1715. - Ae. â zil ō: stone 1046 (: done), goo 1437 (: doo), on 1178 (: John), ropes 1341 (: dropes), more 1370 (: therfore), loo 15S9 (: doo) etc. - Spezifisch nördliche wörter: kirkc 1250, irkc 1252 (vgl. Skeat, Etym. Dict., irk). Hier mag auch erwähnt werden wald (neben wolde 1373 u. ö., nordh. walde) 1919. 1112. 1204 etc. - Nicht dem nördlichen dialekte gehört an bee, part. praet. 1495 (: mee). - Ae. y, umlaut zu u, o gibt regelmässig i (auch y geschrieben): mynd 884. 1316 (: fynd), kind 1003. 1441 (: fynd), syn 1144. 1364 (: in). - Auch sonst wird i begünstigt: z. b. rist, ae. restan, 870, ae. rest 1388; sich 1614 . Dagegen aber resen (part.) 1060, lev(id) 1103. 1496, besiness 1160. 1442. 1450. - Ae.d zeigt sich erhalten in moder, fader, togider, whider, hider; nur 1587 kommt hither vor. - e vor $r$ ist in a übergegangen in marre 1054 (: are), harte 1415 (departe), 1616 (: parte). - Unberechtigtes h: abhominalion 1105, malishosly (maliciously) 891. - Berechtigtes $h$ fehlt: ortulani nach 141i7, armonye 1556. 1620, ympnum (hymnum) nach 1620. - Für $\hat{o}$ wird u geschrieben, z. b. gud 934, blud 1029 (aber bloode 1523), rud 1030 (aber roode 1524, suth 1068. 1069, tuk 1120. 1388; auch burde, ae. bord, 1215. th für $\mathrm{t}$ in comfurth 930. 935. 1012 otc.; dagegen regelmässig comfort 1283. 1426. 1520 etc. - sho neben shee (: mee 918) 971. 1388. - os (neben as 1211 u. o.) 886. 890. 893. - Neben them kommt auch vor tham 1492. 1493. 1538 und thaim 1527. - Part. praet. schwacher verba -t oder -d: blessit 1551, plesit 1315, blessid 1551, levid 111$) 3$ (vgl.comfurth $=$ comforted) 1552 .

Die zahl der hebungen in den versen des ersten teiles wechselt widerum bedeutend; es kommen verse von $1-i$ hebungen vor. Man sieht uberall das bestreben, das zum teil auch durchgefulurt ist, in strophen mit schweifreim die dritte und sechste zeile, in denen in layform die vierte und achte zu kiirzen gegeniiber den anderen. Wol die meisten verse haben vier hebungen, die cäsur, oft durch strich angedeutet, nach der zwoiten, so vv. 4. 6. 12. 14; seltener nach der ersten, z.b. 1. 2. 16; oder cäsurlos, v. 10. Bei fünf hebungen haben wir, wie gewöhnlich, die cäsur nach der zweiten (z. b. 25. 42. 52. 91) oder dritten (so 8. 30. 40. $308-$ wenn thay gestrichen wird; das reimwort zu wrake ist tobrake, praet. plur. zu tobrecen, prädikat zu gret nayles). Sechsmal gehobene verse haben die cäsur gewöhnlich nach der dritten hebung (z.b. 56. 90. 92. 302), auch nach der vierten (so 49. 370) oder zweiten (z. b. 29. 50), selbst nach der ersten (32. 113). Siebenmal gehobene verse haben cïsur nach der vierten hebung (185. 302. 410. 532) oder nach der fünften (370). Verse mit drei hebungen haben die cäsur nach der ersten (31. 39. 58) oder sind cäsurlos (11. 13. 65). Zwei hebungen haben 202. 412. 490. 534. Eine hebung hat nur $\nabla .9$.

Die reime sind gewöhnlich stumpf, nicht selten aber klingend, z. b. sorowe : morowe 56 , render : tender 301 , tender : s'ender 121 etc. Gleiche 
reime kommen selten vor, so $723: 5,79: 84$ u. ö. - Das gedicht zeigt nur wenige verstösse gegen reinheit des reimes, so blisse : giltlesse 659 , malice : excesse 20 , excesse : paleis 21 , attendaunce : reverence 831 , holy : Jesu 863 (oder prosa? als solche gedruckt in Reliquiae Antiquae), son : throng 671, plesinge : hym : thynge 631, Josephe: dethe 14 (vgl. Zupitza zu Guy, Preface XIII). - Andere reime sind leicht rein widerherzustellen. z. b. tawie 3 i (: grave; 'lawie = tame, tractable', Jamieson, Etym. Dict. Scot. Lang. 1882, IV, 518), mother 139 (: othere, vgl. 170), sunder 358, wunder 359, nought 392, glorious 415, vengeaunce 521.

Die strophen bestehen gewöhnlich aus sechszeiligen versen mit der auch in den sammlungen gern gebrauchten schweifreimstellung. Nur selten finden sich abweichungen davon, so durch wideraufnahme der ersten zeile an erster stelle der zweiten hälfte der strophe (aabaab) 508-13. An zusammensetzungen mit jener form haben wir: aabccbddbeeb 307. 422, oder aabaab | ccdaad | cieffe | eegaaag | aaehhe $236 \mathrm{ff}$., in der urspringlichen gestalt, jetzt fehlt ja die zweite hälfte der letzten strophe; ferner aabccb | bbdeed 277; aabcccb | ccbddb 349; aabccb | ccdeed 289; aabccb | ddceec 538. - Auch die in den sammlungen beliebte layform ist angewendet: $16-55.62 \mathrm{ff} .101 \mathrm{ff} .131 \mathrm{ff}$. Damit zusammengesetzt: aaabccbdddeaaae 85 ff.; ferner $a a b a a a b$ | aabcaaab 70 . - Mischung von schweifreim mit layform: aabcccb 200. 271.319. 331.349.416; und in umgekehrter reihenfolge: aaabccb 138 (in urspriinglicher gestalt) 264. 372 (urspriinglich). Aehnlich anabaab 441.378 (urspriinglich); aaabccbddb 109; aabccbdb 823. Ein anderer strophenbau begegnet wider in der achtzeiligen strophe mit der reimstellung $a b a b b c b c$ 1. 622-725; anch hier wideraufnahme des letzten reimes $a b a b b c b c \mid c d c d d e d e$ 622-53; ähnlich ababbcbc | dcdecece | fcfecgcg 662. 694. - Rhymes royal: $726-60.767-87$; verktirzt ababcc 761. - Vierzeilige strophe mit kreuzreim 618. - Drei einreimige zeilen 469. 788. Umschliessender reim mit zwei mittelgliedern 457, mit drei mittelgliedern 9. Reimpaare: 14. 453-6. 467. 598. 791-812. 819-22. 831. 851. 859-64. Reimlos sind 829. 434 (lateinische zeile).

Besonderes interesse in bezug auf die metrik hat dieser teil durch seine refrains in der Marienklage - a) 637. 658. 669.676. 682. 693. 701. 708. 716. 725 ; b) 746. 753. 760; c) 773. 780. 783 - und dadurch, dass sich aus ihm zu dem von Schipper, Altengl. Metrik $§ 317$, mitgeteilten 'unicum' von coblas capfinidas drei strophen, die auch durch concatenatio der einzelnen gebunden sind, anfihren lassen, nämlich z. 694-716.

1 Uebrigens ist Schipper's darstellung a. a. o. dahin zu ergänzen, dass, wenn coblas capfinidas sich auch nur selten weiter durchgeführt finden, sie doch in einzelnen versen öfter vorkommen; z. b. in Town. Myst., Resurrectio Domini, s. 263, Maria Magdalena: Thrughe feetes and handes nayled was hee withouten gyll. Withouten gylt then was he tayn etc. Ib. s. 268, Maria Magdalena: Bol with him spake I bodely, Forthi commen is my blys. My blys is commen etc. Now is he resyn, that was slone, My hart is light. I am as light etc. Ib., Processus talentornm: I have ron fullc fast in hy Hedir to this lowne. To this town now comen am $I$ etc. $\mathrm{Ib}$, Mactatio Abel, Deus: Caym, where is thi brolher Abelle? Caym: What askes thou me? I row at helle. At helle I trow he be. etc. etc. Vgl. auch Horstmann, Altengl. Legenden 18i5, s. XLI, note 4. 
Auch im $z$ weiten teile scheint die hauptmasse der verse vier hebungen zu haben, mit der cäsur nach der zweiten (cïsur ist oft durch einen strich angezeigt), so 875. 977. 927, oder ersten 868. 992. 924, selten fehlend 867. Bei fünf hebungen im verse steht die cäsur nach der zweiten, z. b. 973. 891. 898. 906, oder dritten, so 886. 900, 925, seltener ersten, z. b. 8i0. Der sechsmal gehobene vers hat die cäsur gewöhnlich nach der dritten hebung, so 873 . 874. 889 . 898, selten nach der zweiten, z. b. 906, oder vierten, z. b. 977. Von siebenmal gehobenen versen kommen vor, mit cäsur nach der vierten hebung: 1314. 1335. 1336, nach der dritten: 1177. Nicht selten sind dreihebige verse: 869 . 872. 887 etc. Zweimal gehoben sind 1003. 1208.

Auch hier sind klingende reime nicht selten, so larnentabill : miserabill 879 , stayned : conslrayned 1041 , sorowe : morowe 1259 etc. - Dic reime sind ziemlich rein; erwähnenswert ist nur desire : here 930 , sure : treso(u)re 1517. Wol kaum resurrection : morn 865 (in Reliquiae antiquae als prosa gedruckt). - Einige reime sind leicht widerherzustellen, z. b. alas 932, nought 958 , reservyd 1169 , inquired 1412 , ponderous : gracious 1328, countenance 1559. Ebenso hevy: mercy 923, ye:pcrfitlye 1134, inwertye: ye: dye 1226, trespasse: alasse 1147 (vgl. 1158. 1407), thrîe 1230.

Die mehrzahl der strophen haben auch schweifreimstellung in ihren versen. Weitergefiuhrt ist diese form: $a a b c c b d d b 1569$, aabccbddbeeb 104i, aabccbddbeebddeffe 1265, aabccbccdeedffdfi 1578, aabccbccdeedffd 1473, $a a b c c b d d b d b$ 984. - Layform haben 1380-95. - Mischung von strophen mit schweifreim und in layform aaclccb 1532 und in umgekehrter reihenfolge 1625. - Schweifreimstrophe verziert durch wideraufnahme des reimes der ersten hälfte der strophe in der zweiten: 1253. 1353; damit zusammengesetzt ist aabaabccb 1448. Nicht selten ist diese form in der zweiten hälfte der strophe um eine zeile verkirzt: $a a b a b$ 990. 1065. 1076.1129.1414-53. Oft genug begegnet auch dic form des rhyme royal, z. b. 1283. 1317 . 1320-47. 1359-79, und um die letzte zeile verkïrzt 1225. - Rhyme royal alterniert mit schweifreim 1134-1236. 1595-1620; beide sind durch weitere reime verbunden: $a b a b b c c|a a b d d b| e b e b b f \mid b b g h h g$ 1212; ähnlich, aber regelmässiger: $a b a b b c c|d d c e e c| c f c f f g g$ 1134. - Vierzeilige strophen mit kreuzreimstellung 1249. 1469. 1621. - Reimpare: 1001-10. 1457-64. 1431-4. - Vier einreimige zeilen 1031; fünf einreimige zeilen 1348. - Umschliessender reim aba 1465. - Reimlos 1435. 1466; auch die lateinischen zeilen 1468. 1485. 1501 sind reimlos.

BERLIN.

K. SCHмшт. 